\title{
INTERSECTION FORM, LAMINATIONS AND CURRENTS ON FREE GROUPS
}

\author{
Ilya Kapovich And Martin Lustig
}

\begin{abstract}
Let $F$ be a free group of rank $N \geq 2$, let $\mu$ be a geodesic current on $F$ and let $T$ be an $\mathbb{R}$-tree with a very small isometric action of $F$. We prove that the geometric intersection number $\langle T, \mu\rangle$ is equal to zero if and only if the support of $\mu$ is contained in the dual algebraic lamination $L^{2}(T)$ of $T$. Applying this result, we obtain a generalization of a theorem of Francaviglia regarding length spectrum compactness for currents with full support. We use the main result to obtain "unique ergodicity" type properties for the attracting and repelling fixed points of atoroidal iwip elements of $\operatorname{Out}(F)$ when acting both on the compactified outer Space and on the projectivized space of currents. We also show that the sum of the translation length functions of any two "sufficiently transverse" very small $F$-trees is bilipschitz equivalent to the translation length function of an interior point of the outer space. As another application, we define the notion of a filling element in $F$ and prove that filling elements are "nearly generic" in $F$. We also apply our results to the notion of bounded translation equivalence in free groups.
\end{abstract}

\section{Introduction}

The notion of a geometric intersection number between the free homotopy classes of two essential closed curves on a compact surface plays a fundamental role in the study of the Teichmüller space and of the mapping class group. This notion naturally extends to the notion of an intersection number between a closed curve and a measured lamination (Thurston) as well as the notion of an intersection form between two geodesic currents on a closed hyperbolic surface (Bonahon). The space of measured laminations naturally embeds into the space of currents. Thus Bonahon's intersection form can be used to define the intersection number between a measured lamination (or equivalently, its dual $\mathbb{R}$-tree) and a geodesic current on a surface.

In the free group case, Culler-Vogtmann's outer space $[\mathrm{CuV}], c v(F)$, provides a natural analogue of the Teichmüller space of a surface. The points of $c v(F)$ can be thought of as minimal free discrete actions of $F$ on $\mathbb{R}$-trees. One also often considers the projectivized Outer space $\mathbb{P} c v(F)$ that can be thought of as a subset $C V(F) \subseteq c v(F)$ corresponding to actions where the quotient graph has

Keywords and phrases: Free groups, Outer space, geodesic currents

2000 Mathematics Subject Classification: Primary 20F, Secondary 57M, 37B, 37D

The first author was supported by the NSF grants DMS-0603921 and DMS-0904200. 
volume 1. Taking projective classes of equivariant Gromov-Hausdorff limits of elements of $c v(F)$ leads to a natural Thurston-type compactification $\overline{C V}(F)=$ $C V(F) \cup \partial C V(F)$. The space $\overline{C V}(F)$ turns out to consist precisely of all the projective classes of all minimal very small isometric actions of $F$ on $\mathbb{R}$-trees [BeF3], [CoL]. Recall that an isometric action of $F$ on an $\mathbb{R}$-tree is very small if nontrivial stabilizers of non-degenerate arcs are maximal cyclic and if tripod stabilizers are trivial.

Most automorphisms of $F$ (where $N \geq 3$ ) are not geometric, in the sense that they are not induced by a self-homeomorphism of compact surface with boundary. This leads to the breakdown, in the case of a free group, of numerous symmetries and dualities from the hyperbolic surface situation, and most equivalent notions from the world of surfaces lead to distinct concepts in the free group setting.

The notion of a geodesic current on $F$ (see Definition 2.4 below), and more generally, on a word-hyperbolic group, is a measure-theoretic generalization of the notion of a conjugacy class of a group element or of a free homotopy class of a closed curve on a surface. Much of the motivation for studying currents comes from the work of Bonahon about geodesic currents on hyperbolic surfaces [Bo1,2].

The space $\operatorname{Curr}(F)$ of all geodesic currents has a useful linear structure and admits a canonical $\operatorname{Out}(F)$-action. The space $\operatorname{Curr}(F)$ turns out to be a natural companion of the outer space and contains additional valuable information about the geometry and dynamics of free group automorphisms. Examples of such applications can be found in [Bo3], [CouHL3], [F], [Ka3,4,5], [KaLu1], [KaN], [KKS], [M] and other sources. Kapovich proved [Ka4] that for $F$ there does not exist a natural symmetric analogue of Bonahon's intersection number between two geodesic currents. However, there exists a natural $\operatorname{Out}(F)$-equivariant continuous intersection form

$$
\langle,\rangle: \overline{c v}(F) \times \operatorname{Curr}(F) \rightarrow \mathbb{R},
$$

where $\overline{c v}(F)$ is the space of all very small minimal isometric actions of $F$ on $\mathbb{R}$ trees and where $\operatorname{Curr}(F)$ is the space of geodesic currents on $F$. This intersection form has several important features in common with Bonahon's construction. In particular, if $T \in \overline{c v}(F)$ and $\eta_{g} \in \operatorname{Curr}(F)$ is the counting current for $g \in F-\{1\}$ (see Definition 2.7 below), then

$$
\left\langle T, \eta_{g}\right\rangle=\|g\|_{T},
$$

where $\|g\|_{T}$ is the translation length of $g$ with respect to the tree $T$, that is $\|g\|_{T}=$ $\min _{x \in T} d(x, g x)$. The intersection form was introduced in $[\mathrm{Ka} 3,4],[\mathrm{Lu}]$ for free simplicial actions of $F$, that is for the non-projectivized outer space $c v(F)$. In a recent paper $[\mathrm{KaLu} 2]$ we proved that the intersection form extends continuously to the closure $\overline{c v}(F)$ of $c v(F)$ consisting of all minimal very small isometric actions of $F$ on $\mathbb{R}$-trees. Note that the projectivization $\mathbb{P} \overline{c v}(F)$ of $\overline{c v}(F)$ is exactly the compactification $\overline{C V}(F)$ of $C V(F)$. For $T \in \overline{c v}(F)$ and for $\mu \in \operatorname{Curr}(F)$ we will also call $\langle T, \mu\rangle$ and the geometric intersection number of $T$ and $\mu$.

In general, if $T \in \overline{c v}(F)$ and if $\mu \in \operatorname{Curr}(F)$ is approximated by rational currents as $\mu=\lim _{i \rightarrow \infty} \lambda_{i} \eta_{g_{i}}$, where $g_{i} \in F, \lambda_{i} \geq 0$, the geometric intersection number $\langle T, \mu\rangle$ 
can be computed as

$$
\langle T, \mu\rangle=\lim _{i \rightarrow \infty} \lambda_{i}\left\|g_{i}\right\|_{T}
$$

Ursula Hamenstädt $[\mathrm{H}]$ recently used our result from [KaLu2] about the continuous extension of the intersection form to $\overline{c v}(F)$ as a key ingredient to prove that any non-elementary subgroup of $\operatorname{Out}(F)$, where $N \geq 3$, has infinite-dimensional second bounded cohomology group (infinite-dimensional space of quasi-morphisms). This in turn has an application to proving that any homomorphism from any lattice in a higher-rank semi-simple Lie group to $\operatorname{Out}(F)$, where $N \geq 3$, has finite image.

Very recently Bestvina and Feighn $[\mathrm{BeF} 2]$ used $[\mathrm{KaLu} 2]$ to show that for any finite collection $\phi_{1}, \ldots, \phi_{m} \in \operatorname{Out}\left(F_{N}\right)$ of iwip outer automorphisms of $F_{N}$ ("irreducible automorphisms with irreducible powers", see Definition 12.1) there exists a $\delta$-hyperbolic complex $X=X\left(\phi_{1}, \ldots, \phi_{m}\right)$ with an isometric $\operatorname{Out}\left(F_{N}\right)$-action where each $\phi_{i}$ acts with a positive asymptotic translation length.

Another crucial notion in the surface theory is that of a geodesic lamination on a hyperbolic surface. In the free group case, there is a companion notion of an abstract algebraic lamination which is understood as a closed $F$-invariant and flip-invariant subset of

$$
\partial^{2} F=\left\{\left(\xi_{1}, \xi_{2}\right): \xi_{1}, \xi_{2} \in \partial F, \xi_{1} \neq \xi_{2}\right\} .
$$

A variation of this concept was successfully exploited by Bestvina, Feighn and Handel [BeFH1] to analyze the dynamics of free group automorphisms and the algebraic structure of subgroups of $\operatorname{Out}(F)$. That paper in turn played a key role in the eventual proof of the Tits alternative for $\operatorname{Out}(F)$ by Bestvina, Feighn and Handel in $[\mathrm{BeFH} 2,3]$.

Recently Coulbois, Hilion and Lustig [CouHL1,2,3] gave a detailed abstract treatment of the notion of an algebraic lamination for free group. In particular, given a very small action of $F$ on an $\mathbb{R}$-tree $T$, there is [CouHL2] a naturally defined "dual lamination" $L^{2}(T)$ on $F$ (see section 3 below). In the special case where $T$ belongs to $c v(F)$, i.e. the $F$-action on $T$ is free and simplicial, then $L^{2}(T)=\emptyset$. The relationship between $\mathbb{R}$-tree actions, laminations and geodesic currents in the free group case turns out to be considerably more delicate and complicated then for the case of hyperbolic surfaces. Investigating this relationship is an important basic task in the study of $\operatorname{Out}(F)$.

In the present paper we study the situation where the intersection number between a tree and a current is equal to zero. Our main result is

Theorem 1.1. Let $F$ be a finitely generated nonabelian free group with a very small minimal isometric action on an $\mathbb{R}$-tree $T$. Let $\mu \in \operatorname{Curr}(F)$.

Then $\langle T, \mu\rangle=0$ if and only if $\operatorname{supp}(\mu) \subseteq L^{2}(T)$.

Here $\operatorname{supp}(\mu) \subseteq \partial^{2} F$ is the support of $\mu$ (see section 3 below for the definition).

There is a known similar statement to Theorem 1.1 in the surface context. Namely, suppose $\lambda$ and $\mu$ are measured geodesic laminations on a punctured hyperbolic surface $S$. The transverse measures on $\lambda$ and $\mu$ determine geodesic currents $\hat{\lambda}$ and $\hat{\mu}$ on the surface $S$. In this case Bonahon's intersection number between geodesic currents $\widehat{\lambda}$ coincides with Thurston's geometric intersection number: 
$i(\widehat{\lambda}, \widehat{\mu})=i(\lambda, \mu)$. Moreover, since $\pi_{1}(S)$ is free, geodesic currents on $S$ in Bonahon's sense are also geodesic currents on $\pi_{1}(S)$ in the sense of the present paper. Then $i(\lambda, \mu)=0$ if and only if the supports $\operatorname{supp}(\lambda)$ and $\operatorname{supp}(\lambda)$ of $\lambda$ and $\mu$ intersect in a common sublamination. If $T_{\lambda}$ denotes the "dual" $\mathbb{R}$-tree transverse to $\lambda$ with metric defined by the transverse measure on $\lambda$ (see Ch.11.12 in [Kap] for details), then the definition in [KaLu2] gives

$$
\left\langle T_{\lambda}, \widehat{\mu}\right\rangle=i(\lambda, \mu)=i(\widehat{\lambda}, \widehat{\mu}) .
$$

The lamination $L^{2}\left(T_{\lambda}\right)$ always contains $\operatorname{supp}(\lambda)$, and if the latter fills the surface (i.e. all complementary components are contractible), $L^{2}\left(T_{\lambda}\right)$ is precisely equal to the union of $\operatorname{supp}(\lambda)$ with the (finite) set of diagonal leaves in the complementary surfaces of $\operatorname{supp}(\lambda)$. In particular we see that in this case one has $\left\langle T_{\lambda}, \mu\right\rangle=0$ if and only if $\operatorname{supp}(\mu)$ is contained in $\operatorname{supp}(\lambda) \subseteq \operatorname{supp}\left(T_{\lambda}\right)$.

One of the main motivations and prospective uses for Theorem 1.1 is to analyze the intersection graph, introduced by the authors in [KaLu2] in order to study various free group analogues of the curve complex. The intersection graph $\mathcal{I}(F)$ is a bipartite graph with the vertex set $\mathbb{P} \overline{c v}(F) \sqcup \mathbb{P} \operatorname{Curr}(F)$ where $[T] \in \mathbb{P} \overline{c v}(F)$ and $[\mu] \in \mathbb{P} \operatorname{Curr}(F)$ are adjacent in $\mathcal{I}(F)$ if and only if $\langle T, \mu\rangle=0$. While it is generally not possible to define a good notion of an intersection number between two conjugacy classes in a free group, one can use the intersection form to generalize the notion of having distance $\leq 2$ in the standard curve complex. Thus one can define a graph, whose vertices are conjugacy classes of primitive elements in $F$ where two vertices $[a],[b]$ are adjacent if there exists $T \in \overline{c v}(F)$ such that $\left\langle T, \eta_{a}\right\rangle=\left\langle T, \eta_{b}\right\rangle=0$, that is $\|a\|_{T}=\|b\|_{T}=0$ (this graph is almost the same as the "dual cut graph" defined in [KaLu2]). Taking a dual point of view, one can think of an essential simple closed curve on a surface as a splitting of the surface group over $\mathbb{Z}$, where two curves are adjacent in the curve complex if and only if the corresponding $\mathbb{Z}$-splittings have a common refinement. This leads to the notion [KaLu2] of a cut graph for $F$ whose vertices are nontrivial splittings of $F$ as the fundamental group of a graph of groups with a single edge and the trivial edge group, and where adjacency again corresponds to having a common refinement. A variation of this construction would declare two splittings to be adjacent if there exists a nontrivial element in $F$ which is elliptic with respect to both of them. All these (as others) natural analogues of the curve complex can be studied by means of the intersection graph. In [KaLu2] we prove that for $N \geq 3$ the intersection graph and all the free group analogues of the curve complex derived from it have infinite diameter, by analyzing the action of iwip automorphisms (see Definition 12.1). Iwip automorphisms are also sometimes referred to as fully irreducible in the literature. Note that Theorem 1.1 gives a characterization of adjacency in the intersection graph.

In this paper we apply Theorem 1.1 to obtain a generalization of a result of Francaviglia $[\mathrm{F}]$ about length spectrum compactness for uniform currents on $F$ (see Definition 2.12 below for the definition of the uniform current with respect to a free basis of $F)$. For $T \in c v(F)$ and $\mu \in \operatorname{Curr}(F)$, the automorphic length spectrum of $\eta$ 
with respect to $T$ is the set

$$
\mathcal{S}_{T}(\mu):=\{\langle T, \phi \mu\rangle: \phi \in \operatorname{Out}(F)\}=\{\langle\phi T, \mu\rangle: \phi \in \operatorname{Out}(F)\} \subseteq \mathbb{R} .
$$

If $T=X(F, A)$ is the Cayley graph of $F$ corresponding to a free basis $A$ of $F$ and $\mu=\nu_{A}$ is the uniform current corresponding to $A$ then

$$
\mathcal{S}_{T}(\mu)=\left\{\lambda_{A}(\phi): \phi \in \operatorname{Out}(F)\right\}
$$

where $\lambda_{A}(\phi)$ is the generic stretching factor of $\phi$ with respect to $A$ (see [KKS], [Ka4] for definitions). Here we obtain

Theorem 1.2. Let $\mu \in \operatorname{Curr}(F)$ be a current with full support and let $T \in c v(F)$. Then,

(1) For any $C>0$ the set

$$
\{\phi \in \operatorname{Out}(F):\langle T, \phi \mu\rangle \leq C\}
$$

is finite.

(2) The set $\mathcal{S}_{T}(\mu)$ is a discrete subset of $\mathbb{R}_{\geq 0}$.

(3) Suppose $\phi_{n} \in \operatorname{Out}(F)$ is an infinite sequence of distinct elements such that for some $\lambda_{n} \geq 0$ and some $\mu^{\prime} \in \operatorname{Curr}(F)$ we have $\lim _{n \rightarrow \infty} \lambda_{n} \phi_{n} \mu=\mu^{\prime}$. Then $\lim _{n \rightarrow \infty} \lambda_{n}=0$.

Francaviglia $[\mathrm{F}]$, using very different methods, established Theorem 1.2 for a certain class of currents with full support, including uniform currents corresponding to free bases of $F$ and, more generally, Patterson-Sullivan currents corresponding to points of $c v(F)$ (see [KaN] for definitions). Theorem 1.2 has applications to the "ideal" version of the Whitehead algorithm for geodesic currents, as explained in [Ka5]. Note that for $T \in c v(F)$ and $\mu \in \operatorname{Curr}(F)$ that does not have full support, the automorphic length spectrum $\mathcal{S}_{T}(\mu)$ need not be discrete. For example, if $\phi \in \operatorname{Out}(F)$ is an iwip (irreducible, with irreducible powers) which is atoroidal (that is, with no periodic conjugacy classes) then there exist $\lambda>1$ and a nonzero "eigencurrent" $\eta \in \operatorname{Curr}(F)$ such that $\phi \eta=\lambda \eta$ (see $[\mathrm{M}]$ ). Then $\phi^{-n} \eta=\frac{1}{\lambda^{n}} \eta$, so that for any $T \in c v(F)$ we have

$$
\left\langle T, \phi^{-n} \eta\right\rangle=\frac{1}{\lambda^{n}}\langle T, \eta\rangle \stackrel{n \rightarrow \infty}{\longrightarrow} 0,
$$

and hence $\mathcal{S}_{T}(\eta)$ is not discrete. A recent paper of R. Sharp [Sh] studies other dynamic-theoretic aspects related to generic stretching factors of free group automorphisms. A new paper of D. Calegari and K. Fujiwara [CF] investigates generalizations of generic stretching factors for different word metrics on word-hyperbolic groups.

We apply Theorem 1.1 to establish "unique ergodicity" type properties for the attracting and repelling fixed points of atoroidal elements of $\operatorname{Out}(F)$ in the compactified outer space and in the projectivized space of currents.

If $\phi \in \operatorname{Out}(F)$ is an atoroidal iwip, then the (left) action of $\phi$ on $\overline{C V}(F)$ has exactly two distinct fixed points, an attracting fixed point $\left[T_{+}\right]$and a repelling fixed point $\left[T_{-}\right]$and similarly, the left action of $\phi$ on $\mathbb{P} \operatorname{Curr}(F)$ has exactly two distinct fixed points, an attracting fixed point $\left[\mu_{+}\right]$and a repelling fixed point $\left[\mu_{-}\right]$. In both cases every point distinct from the two fixed points lies on a "North-South" orbit 
with positive powers of $\phi$ making it converge to the attracting fixed point and with negative powers of $\phi$ making it converge to the repelling fixed point. These facts were established by Levitt and Lustig [LevL] for the compactified outer space $\overline{C V}(F)$ and by Reiner Martin $[\mathrm{M}]$ for $\mathbb{P} \operatorname{Curr}(F)$.

In section 12 we apply Theorem 1.1 to establish unique-ergodicity type statements for $T_{+}$and $\mu_{+}$. These results are summarized in the following:

Theorem 1.3. Let $\phi \in \operatorname{Out}(F)$, where $N \geq 3$, be an atoroidal iwip. Let $\left[T_{+}\right] \in \overline{C V}(F)$ and $\left[\mu_{+}\right] \in \mathbb{P} \operatorname{Curr}(F)$ be the attracting fixed points for the (left) actions of $\phi$ on $\overline{C V}(F)$ and $\mathbb{P} \operatorname{Curr}(F)$ accordingly. Then,

(1) If $[\mu] \in \mathbb{P} \operatorname{Curr}(F)$ is such that $\operatorname{supp}(\mu) \subseteq \operatorname{supp}\left(\mu_{+}\right)$then $[\mu]=\left[\mu_{+}\right]$.

(2) If $[T] \in \overline{C V}(F)$ is such that $L^{2}\left(T_{+}\right) \subseteq L^{2}(T)$ then $[T]=\left[T_{+}\right]$.

(3) Let $[\mu] \in \mathbb{P} \operatorname{Curr}(F)$. Then $\left\langle T_{+}, \mu\right\rangle=0$ if and only if $[\mu]=\left[\mu_{+}\right]$.

(4) Let $[T] \in \overline{C V}(F)$. Then $\left\langle T, \mu_{+}\right\rangle=0$ if and only if $[T]=\left[T_{+}\right]$.

We apply Theorem 1.1 together with Theorem 1.3 to show that for two "sufficiently transverse" trees from $\overline{c v}(F)$ the sum of their translation length functions is bilipschitz equivalent to one coming from an interior point of $c v(F)$. For two functions $f_{1}, f_{2}: F \rightarrow \mathbb{R}_{\geq 0}$ we write $f_{1} \sim f_{2}$ if there exists $C \geq 1$ such that for every $w \in F$ we have

$$
\frac{1}{C} f_{1}(w) \leq f_{2}(w) \leq C f_{1}(w)
$$

We prove

Theorem 1.4. Let $T_{1}, T_{2} \in \overline{c v}(F)$ be such that there does not exist $\mu \in \operatorname{Curr}(F)$, $\mu \neq 0$ such that $\left\langle T_{1}, \mu\right\rangle=\left\langle T_{2}, \mu\right\rangle=0$. Then for every $T \in c v(F)$ we have $\|\cdot\|_{T_{1}}+\|\cdot\|_{T_{2}} \sim\|\cdot\|_{T}$.

Proof. Let $T \in c v(F)$ be arbitrary. Then for any nonzero $\mu \in \operatorname{Curr}(F)$ we have $\langle T, \mu\rangle>0$. This follows from the explicit definition of the intersection form in Proposition-Definition 2.11 for the case where $T \in c v(F)$.

Define a function $\mathcal{J}: \operatorname{Curr}(F)-\{0\} \rightarrow \mathbb{R}$ as

$$
\mathcal{J}(\mu):=\frac{\left\langle T_{1}, \mu\right\rangle+\left\langle T_{2}, \mu\right\rangle}{\langle T, \mu\rangle}, \quad \mu \in \operatorname{Curr}(F), \mu \neq 0 .
$$

Note that for every $\mu \in \operatorname{Curr}(F), \mu \neq 0$, we have $0<\mathcal{J}(\mu)<\infty$ by assumption on $T_{1}, T_{2}$. Also, the function $\mathcal{J}$ is continuous by construction since the intersection form is continuous. Moreover, it is easy to see that for every $\mu \neq 0$ and every $c>0$ we have $\mathcal{J}(\mu)=\mathcal{J}(c \mu)$. Thus $\mathcal{J}$ factors through to a continuous strictly positive function $\mathcal{J}^{\prime}: \mathbb{P} \operatorname{Curr}(F) \rightarrow \mathbb{R}$. Since $\mathbb{P} \operatorname{Curr}(F)$ is compact, the function $\mathcal{J}^{\prime}$ achieves a positive maximum and a positive minimum. Hence there exist $0<C_{1}<C_{2}<\infty$ such that $C_{1} \leq \mathcal{J}(\mu) \leq C_{2}$ for every $\mu \in \operatorname{Curr}(F), \mu \neq 0$. Applying this fact to rational currents $\eta_{g}, g \in F-\{1\}$ we conclude that $\|\cdot\|_{T_{1}}+\|\cdot\|_{T_{2}} \sim\|\cdot\|_{T}$, as required.

In the terminology of [KaLu2], the assumption of Theorem 1.4 says that the distance between $\left[T_{1}\right]$ and $\left[T_{2}\right]$ in the intersection graph $\mathcal{I}(F)$ is bigger than two. Using Theorem 1.1 together with Theorem 1.3 we obtain the following corollary of Theorem 1.4. 
Corollary 1.5. Let $N \geq 2$. Then the following hold:

(1) Let $T_{1}, T_{2} \in \overline{c v}(F)$ be such that $L^{2}\left(T_{1}\right) \cap L^{2}\left(T_{2}\right)=\emptyset$. Then for any $T \in c v(F)$ we have $\|\cdot\|_{T_{1}}+\|\cdot\|_{T_{2}} \sim\|\cdot\|_{T}$.

(2) Let $N \geq 3$ and let $\phi \in \operatorname{Out}(F)$ be an atoroidal iwip. Let $\left[T_{+}\right],\left[T_{-}\right] \in \overline{C V}(F)$ be the attracting and repelling fixed points of $\phi$. Then for every $T \in c v(F)$ we have $\|\cdot\|_{T_{+}}+\|\cdot\|_{T_{-}} \sim\|\cdot\|_{T}$.

(3) Let $N \geq 3$ and let $\phi, \psi \in \operatorname{Out}(F)$ be atoroidal iwips such that their fixed points $\left[T_{ \pm}(\phi)\right],\left[T_{ \pm}(\psi)\right] \in \overline{C V}(F)$ are four distinct points. Then for every $T \in c v(F)$ we have $\|\cdot\|_{T_{+}(\phi)}+\|\cdot\|_{T_{+}(\psi)} \sim\|\cdot\|_{T}$.

(4) Let $T_{1}, T_{2} \in \overline{c v}(F)$ be discrete simplicial trees with trivial arc stabilizers (thus, algebraically, they correspond to graph of groups decompositions of $F$ with trivial edge groups). Suppose that every nontrivial elliptic element for $T_{1}$ is hyperbolic for $T_{2}$ and that every nontrivial elliptic element for $T_{2}$ is hyperbolic for $T_{1}$ (that is the intersection of every conjugate of a vertex group of $T_{1}$ with every conjugate of a vertex group of $T_{2}$ is trivial). Then for any $T \in c v(F)$ we have $\|\cdot\|_{T_{1}}+\|\cdot\|_{T_{2}} \sim\|\cdot\|_{T}$.

Proof. Part (1) follows directly from Theorem 1.1 together with Theorem 1.4. Parts (2) and (3) easily follow from Theorem 1.4 together with Theorem 1.1 and Theorem 1.3. We will give an argument for part (3) for concreteness and leave part (2) to the reader. The assumptions of part (3) together with Theorem 1.3 imply that the fixed points $\left[\mu_{ \pm}(\phi)\right],\left[\mu_{ \pm}(\psi)\right]$ of $\phi, \psi$ in $\mathbb{P} \operatorname{Curr}(F)$ are four distinct points. Hence, again, by Theorem 1.3, there does not exist $\mu \neq 0$ such that $\left\langle T_{+}(\phi), \mu\right\rangle=\left\langle T_{+}(\psi), \mu\right\rangle=0$. Hence by part (1) the conclusion of part (3) follows.

To see that part (4) holds it is not hard to show, using the explicit description of $L^{2}\left(T_{1}\right)$ and $L^{2}\left(T_{2}\right)$ obtained in Lemma 8.2 that under the assumptions of part (4) we have $L^{2}\left(T_{1}\right) \cap L^{2}\left(T_{2}\right)=\emptyset$. Hence part (1) of the corollary applies.

Theorem 1.1 has some interesting applications to the notions of a filling element (or a conjugacy class) and of bounded translation equivalence. The concept of a filling curve on a surface plays an important role in the surface theory. However, until now there was no clear analogue of this notion in the free group context, mainly because of the absence of a symmetric notion of an intersection number between two conjugacy classes (or between two currents) for a free group. We propose a natural asymmetric notion of a filling conjugacy class here. It is easy to see that the free homotopy class of an essential closed curve on a closed hyperbolic surface fills the surface if and only if this class has a positive intersection number with every measured lamination on the surface, or equivalently, if and only if the corresponding element of the surface group has positive translation length for the dual $\mathbb{R}$-tree of every measured lamination. By analogy, we will say that an element $g \in F, g \neq 1$ fills $F$ if for every very small isometric action of $F$ on an $\mathbb{R}$-tree $T$ we have $\|g\|_{T}>0$. More generally, we say that a current $\mu \in \operatorname{Curr}(F)$ fills $F$ if for every very small isometric action of $F$ on an $\mathbb{R}$-tree $T$ we have $\langle T, \mu\rangle>0$. Thus an element $g \in F$ fills $F$ if and only if the corresponding "counting current" $\eta_{g} \in \operatorname{Curr}(F)$ fills $F$. As was explained to the authors by Vincent Guirardel, the results of his paper [Gu] can 
be used to show that an element $g \in F$ fills $F$ if and only if for every very small simplicial action of $F$ on an $\mathbb{R}$-tree $T$ we have $\|g\|_{T}>0$. However, we do not use this fact in this paper and work directly with the definition of a filling element given above.

Unlike in the surface case, it is not at all obvious that in a free group $F$ filling elements exist and even less clear why being filling is a typical behavior. In fact, there is no known simple (or even complicated) explicit combinatorial criterion which guarantees that a given element of a free group is filling. Vincent Guirardel and Gilbert Levitt showed us a special construction for producing filling elements in free groups using iterated commutators and high powers. However, one would still like to understand why being filling is an essentially generic property for elements of free groups. Theorem 1.1 provides such an explanation. This theorem easily implies that every current on $F$ with full support fills $F$. While counting currents of elements of $F$ never have full support, it turns out that any multiple of a counting current that is "sufficiently close" to a current with full support does fill $F$ :

Corollary 1.6. Let $F$ be a finitely generated nonabelian free group. Let $\mu \in \operatorname{Curr}(F)$ be a current with full support. Then

(1) The current $\mu$ fills $F$.

(2) Suppose $g_{i} \in F, \lambda_{i} \geq 0$ are such that

$$
\mu=\lim _{i \rightarrow \infty} \lambda_{i} \eta_{g_{i}} .
$$

Then there is some $i_{0} \geq 1$ such that for every $i \geq i_{0}$ the element $g_{i}$ fills $F$.

Note that rational currents (i.e. scalar multiples of counting currents) are dense in $\operatorname{Curr}(F)$, so that any current $\mu$ with full support can be approximated by rational currents. Corollary 1.6 in turn implies that if $A$ is a free basis of $F$ and $\xi$ is a random right-infinite freely reduced word over $A^{ \pm 1}$, then all sufficiently long initial segments of $\xi$ give filling elements in $F$ :

Corollary 1.7. Let $F=F(A)$ be a finitely generated nonabelian free group with a free basis $A$. Let $\mu_{A}$ be the uniform measure on $\partial F$ corresponding to $A$. Then there exists a set $R \subseteq \partial F$ with the following properties:

(1) We have $\mu_{A}(R)=1$.

(2) For each $\xi \in R$ there is $n_{0} \geq 1$ such that for every $n \geq n_{0}$ the element $\xi_{A}(n) \in F$ fills $F$.

Here $\xi_{A}(n) \in F$ denotes the element of $F$ corresponding to the initial segment of $\xi$ of length $n$. The uniform measure $\mu_{A}$ on $\partial F$ is a Borel probability measure (see Definition 13.5 for a precise definition), such that a $\mu_{A}$-random point of $\partial F$ corresponds to the intuitive notion of a "random" right-infinite freely reduced word over $A$.

In [KaLSS], Kapovich, Levitt, Schupp and Shpilrain introduced the notion of translation equivalence in free groups, motivated by the notions of hyperbolic equivalence and simple intersection equivalence for curves in surfaces (see [L]). Recall that two elements $g, h \in F$ are translation equivalent in $F$ if for every very small action of $F$ on an $\mathbb{R}$-tree $T$ we have $\|g\|_{T}=\|h\|_{T}$. The paper [KaLSS] exhibited 
several sources of translation equivalence and additional ones were found by Donghi Lee [Le]. The following is a natural generalization of the notion of translation equivalence. We say that $g, h \in F$ are boundedly translation equivalent in $F$, denoted $g \equiv_{b} h$, if there exists $C>0$ such that for for every very small action of $F$ on an $\mathbb{R}$-tree $T$ we have

$$
\frac{1}{C}\|h\|_{T} \leq\|g\|_{T} \leq C\|h\|_{T} .
$$

The following statement, together with Corollary 1.6 and Corollary 1.7, explains why, unlike translation equivalence, bounded translation equivalence is an essentially generic phenomenon in free groups.

Corollary 1.8. Let $g, h \in F$ be elements such that each of them fills $F$. Then $g \equiv_{b} h$ in $F$.

Note that while Corollary 1.6 and Corollary 1.7 do explain why filling elements in $F$ are plentiful, they do not provide an explicit sufficient condition for an element to be filling. Finding such a sufficient condition that would be easily algorithmically verifiable and that would hold for "generic" elements of $F$ remains an interesting problem. The construction of Guirardel and Levitt mentioned above is both explicit and algorithmic, but it relies on using iterated commutators and large powers and thus is highly non-generic.

The paper is organized as follows. In section 2 we review basic definitions and notation related to outer space and geodesic currents. In section 3 we present background information regarding algebraic laminations on free groups, introduce the notions of the support of a current and the "dual lamination" associated with an action of a free group on an $\mathbb{R}$-tree and establish some basic facts about laminations and subgroups. In section 4 we discuss the "bounded back-tracking" property for very small actions of $F$ on $\mathbb{R}$-trees and its consequences. The bounded back-tracking property for very small actions, established in [GJLL], is a key tool in the present paper. In section 5 we prove the "if" direction of the main result, Theorem 1.1. Namely, in Theorem 5.2 we prove that if $\operatorname{supp}(\mu) \subseteq L^{2}(T)$ then $\langle T, \mu\rangle=0$. The "only if" direction of Theorem 1.1 turns out to be more difficult and requires considering several different types of trees $T$ separately. In section 6 we establish the "only if" direction of Theorem 1.1 for the case of a tree $T \in \overline{c v}(F)$ with dense orbits. The case of a discrete $T \in \overline{c v}(F)$ is dealt with in section 7 and section 8. Before dealing with the general case, we develop some new machinery for restricting geodesic currents to subgroups of $F$ in section 9 . In section 10 we establish the "only if" direction of Theorem 1.1 in the "mixed" or general case of an arbitrary $T \in \overline{c v}(F)$. This is done in Theorem 10.7 which completes the proof of Theorem 1.1. In section 11 we apply the main result to prove Theorem 1.2 about length spectrum compactness (Theorem 11.2 in section 11). In section 12 we establish the unique-ergodicity type results stated in Theorem 1.3 above. In section 13 we obtain applications of Theorem 1.1 to filling elements, filling currents and bounded translation equivalence, stated in Corollary 1.6, Corollary 1.7 and Corollary 1.8 above. 
We are grateful to Vincent Guirardel, Gilbert Levitt, Pascal Hubert and Chris Leininger for helpful comments and conversations. We are also grateful to the referee for an extremely careful and detailed referee report and for numerous useful suggestions.

\section{Geodesic Currents}

Convention 2.1. For the remainder of the article let $F$ be a finitely generated nonabelian free group.

Let $\partial F$ be the hyperbolic boundary of $F$ (see $[\mathrm{GyH}]$ for background information about word-hyperbolic groups). We denote

$$
\partial^{2} F=\left\{\left(\xi_{1}, \xi_{2}\right): \xi_{1}, \xi_{2} \in \partial F \text { and } \xi_{1} \neq \xi_{2}\right\} .
$$

Also denote by $\sigma_{F}: \partial^{2} F \rightarrow \partial^{2} F$ the "flip" map defined as $\sigma_{F}:\left(\xi_{1}, \xi_{2}\right) \mapsto\left(\xi_{2}, \xi_{1}\right)$ for $\left(\xi_{1}, \xi_{2}\right) \in \partial^{2} F$.

Definition 2.2 (Simplicial charts). A simplicial chart on $F$ is an isomorphism $\alpha: F \rightarrow \pi_{1}(\Gamma, x)$ where $\Gamma$ is a finite connected graph without degree-one vertices and where is a vertex of $\Gamma$.

From now on, when discussing graphs, for a graph $\Gamma$ we will denote the set of vertices of $\Gamma$ by $V \Gamma$.

If $\alpha$ is a simplicial chart on $F$, it defines an $F$-equivariant quasi-isometry between $F$ (with any word metric) and $\widetilde{\Gamma}$, with the simplicial metric, that is where every edge has length 1 . Correspondingly, we get canonical $F$-equivariant homeomorphisms $\widetilde{\alpha}: \partial F \rightarrow \partial \widetilde{\Gamma}$ and $\widehat{\alpha}: \partial^{2} F \rightarrow \partial^{2} \widetilde{\Gamma}$, that do not depend on the choice of a word metric for $F$. If $\alpha$ is fixed, we will usually use these homeomorphisms to identify $\partial F$ with $\partial \widetilde{\Gamma}$ and $\partial^{2} F$ with $\partial^{2} \widetilde{\Gamma}$ without additional comment.

Definition 2.3 (Cylinders). Let $\alpha: F \rightarrow \pi_{1}(\Gamma, x)$ be a simplicial chart on $F$. For a nontrivial reduced edge-path $\gamma$ in $\widetilde{\Gamma}$ denote by $\operatorname{Cyl}_{\widetilde{\Gamma}}(\gamma)$ the set of all $\left(\xi_{1}, \xi_{2}\right) \in \partial^{2} F$ such that the bi-infinite geodesic from $\tilde{\alpha}\left(\xi_{1}\right)$ to $\tilde{\alpha}\left(\xi_{2}\right)$ contains $\gamma$ as a subpath.

We call $\operatorname{Cyl}_{\widetilde{\Gamma}}(\gamma) \subseteq \partial^{2} F$ the two-sided cylinder corresponding to $\gamma$.

It is easy to see that $\operatorname{Cyl}_{\widetilde{\Gamma}}(\gamma) \subseteq \partial^{2} F$ is both compact and open. Moreover, the collection of all such cylinders, where $\gamma$ varies over all nontrivial reduced edge-paths in $\widetilde{\Gamma}$, forms a basis of open sets in $\partial^{2} F$.

Definition 2.4 (Geodesic currents). A geodesic current on $F$ is a positive Radon measure (that is a Borel measure which is finite on compact sets) on $\partial^{2} F$ that is $F$-invariant and $\sigma_{F}$-invariant. The set of all geodesic currents on $F$ is denoted by $\operatorname{Curr}(F)$. The set $\operatorname{Curr}(F)$ is endowed with the weak topology which makes it into a locally compact space.

Specifically, let $\alpha: F \rightarrow \pi_{1}(\Gamma, x)$ be a simplicial chart on $F$. Let $\mu_{n}, \mu \in \operatorname{Curr}(F)$. It is not hard to show [Ka4] that $\lim _{n \rightarrow \infty} \mu_{n}=\mu$ in $\operatorname{Curr}(F)$ if and only if for every nontrivial reduced edge-path $\gamma$ in $\widetilde{\Gamma}$ we have

$$
\lim _{n \rightarrow \infty} \mu_{n}\left(\mathrm{Cyl}_{\widetilde{\Gamma}}(\gamma)\right)=\mu\left(\mathrm{Cyl}_{\widetilde{\Gamma}}(\gamma)\right) .
$$


Let $\mu \in \operatorname{Curr}(F)$ and let $v$ be a nontrivial reduced edge-path in $\Gamma$. Denote

$$
\langle v, \mu\rangle_{\alpha}:=\mu\left(\operatorname{Cyl}_{\widetilde{\Gamma}}(\gamma)\right),
$$

where $\gamma$ is any edge-path in $\widetilde{\Gamma}$ that is labelled by $v$ (i.e. which is a lift of $v$ to $\widetilde{\Gamma}$ ). Since $\mu$ is $F$-invariant, this definition does not depend on the choice of a lift $\gamma$ of $v$.

Notation 2.5. Let $A=\left\{a_{1}, \ldots, a_{k}\right\}$ be a free basis of $F$ and let $\alpha$ be the simplicial chart on $F$ corresponding to $A$. That is, $\alpha: F \rightarrow \pi_{1}(\Gamma, x)$, where $\Gamma$ is a wedge of $k$ loop-edges at a single vertex $x$, where the edges are labelled by $a_{1}, \ldots a_{k}$. The map $\alpha$ sends a freely reduced word $v \in F(A)$ to the edge-path in $\Gamma$ labelled by $v$. Then $\widetilde{\Gamma}=X(F, A)$ is the Cayley graph of $F$ with respect to $A$.

In this case, for $v \in F(A)$ and $\mu \in \operatorname{Curr}(F)$, we will denote $\langle\alpha(v), \mu\rangle_{\alpha}$ by $\langle v, \mu\rangle_{A}$. Notation 2.6. For any $g \in F, g \neq 1$ denote $g^{\infty}=\lim _{n \rightarrow \infty} g^{n}$ and $g^{-\infty}=$ $\lim _{n \rightarrow \infty} g^{n}$ so that $\left(g^{-\infty}, g^{\infty}\right) \in \partial^{2} F$.

Also, for any $g \in F$ we will denote by $[g]_{F}$ or just by $[g]$ the conjugacy class of $g$ in $F$.

DEFinition 2.7 (Counting and rational currents). Let $g \in F$ be a nontrivial element that is not a proper power in $F$. Put

$$
\eta_{g}^{F}=\sum_{h \in[g]_{F}}\left(\delta_{\left(h^{-\infty}, h^{\infty}\right)}+\delta_{\left(h^{\infty}, h^{-\infty}\right)}\right) .
$$

Let $\mathcal{R}(g)$ be the collection of all $F$-translates of $\left(g^{-\infty}, g^{\infty}\right)$ and $\left(g^{\infty}, g^{-\infty}\right)$ in $\partial^{2} F$. Note that if $u \in F$ and $h=u g u^{-1}$ then $u\left(g^{-\infty}, g^{\infty}\right)=\left(h^{-\infty}, h^{\infty}\right)$. Therefore

$$
\eta_{g}^{F}=\sum_{(x, y) \in \mathcal{R}(g)} \delta_{(x, y)},
$$

and hence $\eta_{g}^{F}$ is $F$-invariant and flip-invariant, that is $\eta_{g} \in \operatorname{Curr}(F)$.

Let $g \in F$ be an arbitrary nontrivial element. Write $g=f^{m}$ where $m \geq 1$ and $f \in F$ is not a proper power. Put $\eta_{g}^{F}:=m \eta_{f}^{F}$.

We call $\eta_{g}^{F} \in \operatorname{Curr}(F)$ the counting current corresponding to g. Positive scalar multiples of counting currents are called rational currents. If the ambient group $F$ is fixed, we will often denote $\eta_{g}^{F}$ by $\eta_{g}$.

It is easy to see that if $[g]_{F}=[h]_{F}$ then $\eta_{g}^{F}=\eta_{h}^{F}$ and $\eta_{g}^{F}=\eta_{g^{-1}}^{F}$.

The following statement is an important basic fact regarding rational currents.

Proposition $2.8[\mathrm{Ka} 3,4]$. The set of all rational currents is dense in the space $\operatorname{Curr}(F)$.

Definition 2.9 (Cyclic paths and cyclic words). A cyclic path or circuit in $\Gamma$ is an immersion graph-map $c: \mathbb{S} \rightarrow \Gamma$ from a simplicially subdivided oriented circle $\mathbb{S}$ to $\Gamma$. Let $u$ be an edge-path in $\Gamma$. An occurrence of $u$ in $c$ is a vertex of $\mathbb{S}$ such that, going from this vertex in the positive direction along $\mathbb{S}$, there exists an edge-path in $\mathbb{S}$ (not necessarily simple and not necessarily closed) which is labelled by $u$, that is, which is mapped to $u$ by $c$. We denote by $\langle u, c\rangle$ the number of occurrences of $u$ in $c$. 
If $A$ is a free basis of $F$ and $\Gamma$ is a bouquet of edges labelled by the elements of $A$, then a cyclic path in $\Gamma$ can also be thought of as a cyclic word over $A$. A cyclic word is a cyclically reduced word in $F(A)$ written on a simplicially subdivided circle (where every positively oriented edge is labelled by an element of $A$ ) in the clockwise-direction without a specified base-point. The number of occurrences of $v \in F(A)$ in a cyclic word $w$ over $A$ is denoted $\langle v, w\rangle_{A}$.

Let $\alpha: F \rightarrow \pi_{1}(\Gamma, x)$ be a simplicial chart for $F$. Then every nontrivial conjugacy class $[g]_{F}$ is represented by a unique reduced cyclic edge-path $w_{g}$ in $\Gamma$.

If $w$ is a cyclic edge-path in $\Gamma$ and $v$ is an edge-path in $\Gamma$, denote by $\langle v, w\rangle_{\alpha}$ the number of occurrences of $v$ in $w$. We will also occasionally use the notation $\left\langle v^{ \pm 1}, w\right\rangle_{\alpha}:=\langle v, w\rangle_{\alpha}+\left\langle v^{-1}, w\right\rangle_{\alpha}$.

It is not hard to see that the definition of a counting current can be reinterpreted as follows [Ka4]:

Lemma 2.10. Let $\alpha: F \rightarrow \pi_{1}(\Gamma, x)$ be a simplicial chart for $F$. Let $g \in F$ be a nontrivial element and let $w_{g}$ be the reduced cyclic path in $\Gamma$ representing $[g]_{F}$.

Then for every reduced edge-path $v$ in $\Gamma$ we have

$$
\left\langle v, \eta_{g}\right\rangle_{\alpha}=\left\langle v^{-1}, \eta_{g}\right\rangle_{\alpha}=\left\langle v, w_{g}\right\rangle_{\alpha}+\left\langle v^{-1}, w_{g}\right\rangle_{\alpha}=\left\langle v^{ \pm 1}, w\right\rangle_{\alpha} .
$$

Proposition-Definition 2.11 (Intersection form [KaLu2]). Let $F$ be a finitely generated nonabelian free group and with a very small minimal isometric action on an $\mathbb{R}$-tree $T$. Let $\mu \in \operatorname{Curr}(F)$. Let $\mu=\lim _{i \rightarrow \infty} \lambda_{i} \eta_{g_{i}}$ where $\lambda_{i} \geq 0$ and $g_{i} \in F$. Then,

(1) The limit

$$
\lim _{i \rightarrow \infty} \lambda_{i}\left\|g_{i}\right\|_{T}
$$

exists and does not depend on the choice of a sequence of rational currents $\lambda_{i} \eta_{g_{i}}$ approximating $\mu$. We call this limit the length of $\mu$ with respect to $T$ or the geometric intersection number of $T$ and $\mu$ or the length of $\mu$ with respect to $T$ and denote it by $\langle T, \mu\rangle$ or by $\|\mu\|_{T}$.

(2) Let $\alpha: F \rightarrow \pi_{1}(\Gamma, x)$ be a simplicial chart on $F$ and let $\mathcal{L}$ be a metric structure on $\Gamma$. That is, each oriented edge $e \in E \Gamma$ is assigned a length $\mathcal{L}(e)>0$ so that $\mathcal{L}(e)=\mathcal{L}\left(e^{-1}\right)$ for every $e \in E \Gamma$. Let $T$ be the $\mathbb{R}$-tree obtained by giving each edge in $\widetilde{\Gamma}$ the same length as that of its projection in $\Gamma$. Thus $F$ acts on $T$ freely and discretely by isometries, via $\alpha$. Then

$$
\langle T, \mu\rangle=\frac{1}{2} \sum_{e \in E \Gamma} \mathcal{L}(e)\langle e, \mu\rangle_{\alpha}
$$

(3) The function

$$
\langle,\rangle: \overline{c v}(F) \times \operatorname{Curr}(F) \rightarrow \mathbb{R}
$$

is continuous, Out $(F)$-invariant, $\mathbb{R}_{\geq 0}$-linear with respect to the second argument and $\mathbb{R}_{\geq 0}$-homogeneous with respect to the first argument.

Proposition-Definition 2.11 for free simplicial actions was obtained in [Ka4], [Lu]. Recently, Kapovich and Lustig [KaLu2] generalized this result to the case of arbitrary very small actions and proved Proposition-Definition 2.11 in the form stated above. 
Let $A$ be a free basis of $F$ and let $X(F, A)$ be the Cayley graph of $F$ corresponding to $A$. Thus $X(F, A) \in c v(F)$. For a current $\mu \in \operatorname{Curr}(F)$ denote $\|\mu\|_{A}:=\|\mu\|_{X(F, A)}=\langle X(F, A), \mu\rangle$.

Definition 2.12 (Uniform current). Let $A$ be a free basis of $F$ and let $k \geq 2$ be the rank of $F$. Consider the simplicial chart $\alpha$ on $F$ corresponding to $A$. The uniform current corresponding to $A$, denoted by $\nu_{A}$ is the current defined by

$$
\left\langle v, \nu_{A}\right\rangle_{A}=\frac{1}{2 k(2 k-1)^{n-1}},
$$

where $v \in F(A)$ is a nontrivial freely reduced word and where $|v|_{A}=n$.

It is not hard to show that $\nu_{A}$ is indeed a geodesic current on $F$ and we refer the reader to $[\mathrm{Ka} 4]$, $[\mathrm{KKS}]$ for a more detailed discussion.

\section{Laminations}

Recall that, as specified in Convention 2.1, $F$ is a finitely generated nonabelian free group. We refer the reader to [CouHL1,2,3] for detailed background material on algebraic laminations in the context of free groups. We shall only state some basic definitions and facts here.

Definition 3.1 (Algebraic laminations). An algebraic lamination on $F$ is a closed $F$-invariant and flip-invariant subset $L \subseteq \partial^{2} F$.

Denote by $\Lambda^{2}(F)$ the set of all algebraic laminations on $F$.

Definition 3.2 (Laminary language of an algebraic lamination). Let $\alpha: F \rightarrow$ $\pi_{1}(\Gamma, x)$ be a simplicial chart of $F$ and let $X=\widetilde{\Gamma}$. Recall that $\alpha$ defines a canonical $F$-equivariant homeomorphism $\widetilde{\alpha}: \partial F \rightarrow \partial X$

Let $B$ be the set of oriented edges of $\Gamma$. Let $L \in \Lambda^{2}(F)$. The laminary language of $L$, corresponding to $\alpha$, denoted $L_{\alpha}$, is defined as the set of all reduced edgepaths $v$ in $\Gamma$ such that there exists a bi-infinite geodesic $\gamma$ in $X$ with endpoints $\widetilde{\alpha}\left(\xi_{1}\right), \widetilde{\alpha}\left(\xi_{2}\right) \in \partial X$ for some $\left(\xi_{1}, \xi_{2}\right) \in L$, such that $\gamma$ contains a subsegment labelled by $v$. We can think of $L_{\alpha}$ as a subset of $B^{*}$, where $B^{*}$ is the set of all words in the alphabet $B$.

If $\alpha$ is a simplicial chart on $F$ corresponding to a free basis $A$ of $F$ (where $\Gamma$ is the wedge of circles labelled by elements of $A$ and where $X$ is the Cayley graph of $F$ with respect to $A$ ), we will denote $L_{\alpha}$ by $L_{A}$. In this case $L_{A}$ is a set of freely reduced words in $F=F(A)$.

Note that in [CouHL1,2,3] laminary languages are defined only with respect to a free basis of a free group. However, it is easy to see that the definition and the basic results listed here extend to an arbitrary simplicial chart, that is not necessarily a wedge of loop-edges.

Proposition 3.3 [CouHL1]. Let $\alpha: F \rightarrow \pi_{1}(\Gamma, x)$ be a simplicial chart of $F$. Let $L, L^{\prime} \in \Lambda^{2}(F)$. Then $L \subseteq L^{\prime}$ if and only if $L_{\alpha} \subseteq L_{\alpha}^{\prime}$. 
Let $H \leq F$ be a finitely generated subgroup. Then the inclusion of $H$ in $F$ extends to a canonical $H$-equivariant topological embedding $i_{H}: \partial H \rightarrow \partial F$. We will usually suppress this embedding and write that $\partial H \subseteq \partial F$ and $\partial^{2} H \subseteq \partial^{2} F$.

Proposition-Definition 3.4. Let $H \leq F$ be a finitely generated subgroup and let $L \in \Lambda^{2}(H)$ be an algebraic lamination on $H$. Then

$$
i_{\Lambda}(L):=\overline{\bigcup_{f \in F} f L}
$$

is a closed $F$-invariant subset of $\partial^{2} F$, that is, an algebraic lamination on $F$. Thus $i_{\Lambda}(L) \in \Lambda^{2}(F)$.

Definition 3.5 (Lamination defined by a tree action [CouHL1,2,3]). Let $F$ be a finitely generated free group acting isometrically on an $\mathbb{R}$-tree $T$. The lamination on $F$ corresponding to the action of $F$ on $T$, denoted $L^{2}(T) \in \Lambda^{2}(F)$, is defined as follows.

Choose a simplicial chart $\alpha: F \rightarrow \pi_{1}(\Gamma, x)$ and fix the corresponding identification of $\partial^{2} F$ and $\partial^{2} \widetilde{\Gamma}$.

Then for $\left(\xi_{1}, \xi_{2}\right) \in \partial^{2} F$ we have $\left(\xi_{1}, \xi_{2}\right) \in L^{2}(T)$ if and only if, for every $\epsilon>0$ and every reduced edge-path $v$ in $\Gamma$ labelling some subsegment of the bi-infinite geodesic joining $\xi_{1}$ and $x_{2}$ in $\widetilde{\Gamma}$, there is some reduced and cyclically reduced closed path $w$ in $\Gamma$ containing $v$ as a subpath such that $\|w\|_{T} \leq \epsilon$.

It is not hard to show (see [LevL], [CouHL2]) that this definition of $L^{2}(T)$ does not depend on the choice of a simplicial chart on $F$.

Definition 3.6 (Support of a current). Let $\mu \in \operatorname{Curr}(F)$. Then we define the support of $\mu$, denoted $\operatorname{supp}(\mu)$, as $\partial^{2} F-\mathcal{U}$ where $\mathcal{U}$ is the union of all open subsets $U \subseteq \partial^{2} F$ such that $\mu(U)=0$. It is easy to see that $\operatorname{supp}(\mu) \subseteq \partial^{2} F$ is both closed and $F$-invariant, so that $\operatorname{supp}(\mu) \in \Lambda^{2}(F)$. We say that $\mu \in \operatorname{Curr}(F)$ has full support if $\operatorname{supp}(\mu)=\partial^{2} F$.

Let $\alpha: F \rightarrow \pi_{1}(\Gamma, x)$ be a simplicial chart on $F$, let $L=\operatorname{supp}(\mu) \in \Lambda^{2}(F)$ and let $B=E \Gamma$. We will denote the laminary language $L_{\alpha} \subseteq B^{*}$ by $\operatorname{supp}_{\alpha}(\mu)$. Thus $\operatorname{supp}_{\alpha}(\mu)$ consists of all reduced paths $v$ in $\Gamma$ such that $\langle v, \mu\rangle_{\alpha}>0$.

In the case where the simplicial chart $\alpha$ is defined by a free basis $A$ of $F$, we will denote $L_{\alpha}$ by $\operatorname{supp}_{A}(\mu)$.

For example, it is obvious that for any free basis $A$ of $F$ the uniform current $\nu_{A}$ has full support. The following lemma is an easy corollary of the definitions.

Lemma 3.7. Let $\alpha: F \rightarrow \pi_{1}(\Gamma, x)$ be a simplicial chart on $F$ and let $\mu \in \operatorname{Curr}(F)$. Let $L=\operatorname{supp}(\mu) \in \Lambda^{2}(F)$. Then for a reduced edge-path $v$ in $\Gamma$ we have $v \in L_{\alpha}$ if and only if $\langle v, \mu\rangle_{\alpha}>0$.

Notation 3.8. Let $F$ be a finitely generated free group with a free basis $A$. We will denote by $X(F, A)$ the Cayley graph (which happens to be a tree) of $F$ with respect to $A$.

For a nontrivial finitely generated subgroup $H \leq F$ denote by $X_{H}$ the smallest $H$-invariant subtree of $X(F, A)$. 
We also denote by $\Gamma_{H}$ the Stallings subgroup graph of $H$ with respect to $A$ (see $[\mathrm{KaM}]$ for a detailed discussion about Stallings subgroup graphs). Recall that $\Gamma_{H}$ can be obtained as follows. Let $x \in X_{H}$ be the closest to $1 \in F$ vertex of $X_{H}$. Then $X_{H}^{\prime}=X_{H} \bigcup \cup_{f \in F} f[1, x]$ is an $H$-invariant subtree of $X(F, A)$. Then $\Gamma_{H}=X_{H}^{\prime} / H$. Recall that every oriented edge in $\Gamma_{H}$ has a label $a \in A^{ \pm 1}$ which comes from the corresponding label of the edge in $X_{H}^{\prime}$. The image, under the quotient map $X_{H}^{\prime} \rightarrow$ $X_{H}^{\prime} / H=\Gamma_{H}$ of the vertex $1 \in F$ in $\Gamma_{H}$ is the base-vertex of $\Gamma_{H}$.

For a finite connected graph $\Gamma$ with a nontrivial fundamental group, the core of $\Gamma$, denoted Core $(\Gamma)$, is the unique smallest subgraph $\Delta$ of $\Gamma$ such that $\Delta$ is homotopically equivalent to $\Gamma$. Note that $\Delta$ has no degree-one vertices and that $\Gamma$ is equal to the union of $\Delta$ and a finite (possibly empty) collection of trees attached to some vertices of $\Delta$.

Convention 3.9. For the remainder of this section, let $H \leq F=F(A)$ be a finitely generated subgroup. Let $\Gamma_{H}$ be the Stallings subgroup graph of $H$ with respect to $A$ and let $\Delta_{H}=\operatorname{Core}\left(\Gamma_{H}\right)$.

Let $x \in \Delta_{H}$ be the closest vertex of $\Delta_{H}$ to the basepoint $y$ of $\Gamma_{H}$. Let $u \in F(A)$ be the label of the segment joining $y$ to $x$ in $\Gamma_{H}$. Then we have a canonical isomorphism $\alpha_{H, A}: H \rightarrow \pi_{1}\left(\Gamma_{H}, y\right)$, where $\alpha_{H, A}^{-1}$ sends a loop $\gamma$ at $x$ in $\Delta_{H}$ to $u w u^{-1}$, where $w \in F(A)$ is the label of $\gamma$. Thus the pair $\left(\Gamma_{H}, y\right)$ defines a canonical isomorphism $\alpha_{H, A}: H \rightarrow \pi_{1}\left(\Gamma_{H}, y\right)$ which is a simplicial chart for $H$. Moreover, a copy of $\widetilde{\Delta}_{H}$ is contained in $X(F, A)$ and coincides with the smallest $H$-invariant subtree of $X(F, A)$.

The following lemma is easily established by compactness argument since the graph $\Delta_{H}$ is finite.

Lemma 3.10. Let $L \in \Lambda^{2}(H)$ and let $L^{\prime}=i_{\Lambda}(L) \in \Lambda^{2}(F)$. Then for $v \in F(A)$ we have $v \in L_{A}^{\prime}$ if and only if there is a bi-infinite reduced path $\gamma$ in $\Delta_{H}$ whose $F(A)$ label contains $v$ as a subword, such that some bi-infinite geodesic in $\widetilde{\Delta}_{H}$, whose pair of endpoints is an element of $L$, projects to $\gamma$.

Lemma 3.10 in turn easily implies

LEMma 3.11. The following hold:

(1) Let $\left(\xi_{1}, \xi_{2}\right) \in \partial^{2} F$. Then $\left(\xi_{1}, \xi_{2}\right) \in i_{\Lambda}\left(\partial^{2} H\right)$ if and only if the label of the biinfinite geodesic from $\xi_{1}$ to $\xi_{2}$ in $X(F, A)$ is the $F(A)$-label of some bi-infinite reduced edge-path in Core $\left(\Gamma_{H}\right)$.

(2) The subset $\bigcup_{f \in F} f \partial^{2} H \subseteq \partial^{2} F$ is closed, and hence

$$
i_{\Lambda}\left(\partial^{2} H\right)=\bigcup_{f \in F} f \partial^{2} H .
$$

\section{Bounded Back-Tracking}

Definition 4.1 (Bounded back-tracking constant). Let $F=F(A)$ be a finitely generated free group acting isometrically on an $\mathbb{R}$-tree $T$. Let $X(F, A)$ be the Cayley graph of $F$ with respect to $A$. Let $p \in T$. There is a unique $F$-equivariant map 
$i_{p}: X(F, A) \rightarrow T$ that is linear on edges on $X(F, A)$ and with $i_{p}(1)=p$. The bounded back-tracking constant corresponding to $A$, $T$ and $p$, denoted $B B T_{T, p}(A)$, is the infimum of all $C>0$ such that for any $Q, R \in X(F, A)$ the image $i_{p}([Q, R])$ of $[Q, R] \subseteq X(F, A)$ is contained in the $C$-neighborhood of $\left[i_{p}(Q), i_{p}(R)\right]$. The bounded back-tracking constant corresponding to $A$ and $T$, denoted $B B T_{T}(A)$, is the infimum over all $p \in T$ of $B B T_{T, p}(A)$.

An useful result of [GJLL] states

Proposition 4.2. Let $F$ be a finitely generated nonabelian free group with a very small isometric minimal action on an $\mathbb{R}$-tree $T$. Let $A$ be a free basis of $F$ and let $p \in T$. Then $B B T_{T, p}(A)<\infty$.

The following is an easy corollary of the definitions.

LEMma 4.3. Let $F$ be a finitely generated nonabelian free group with a very small isometric minimal action on an $\mathbb{R}$-tree $T$. Let $A$ be a free basis of $F$ and let $p \in T$.

Suppose $B B T_{T, p}(A)<C$. Let $u=u_{1} \ldots u_{m}$ be a freely reduced product of freely reduced words in $F(A)$, where $m \geq 1$. Then the following hold:

(1) Let $w \in F(A)$ be cyclically reduced. Then

$$
\left|\|w\|_{T}-d_{T}(p, w p)\right| \leq 2 C .
$$

(2) Let $u=u_{1} \ldots u_{m}$ be a freely reduced product of freely reduced words in $F=F(A)$, where $m \geq 1$. Then we have

$$
\left|d_{T}(p, u p)-\sum_{i=1}^{m} d_{T}\left(p, u_{i} p\right)\right| \leq 2 m C .
$$

(3) Suppose, in addition, that $u, u_{1}, \ldots, u_{m}$ are cyclically reduced in $F(B)$. Then

$$
\left|\|u\|_{T}-\sum_{i=1}^{m}\left\|u_{i}\right\|_{T}\right| \leq 4 m C .
$$

Levitt and Lustig [LevL] prove the following:

Proposition 4.4. Let $F$ be a finitely generated nonabelian free group with a very small isometric action on an $\mathbb{R}$-tree $T$ with dense orbits.

Then for any $\epsilon>0$ and any $p \in T$ there exists a free basis $B$ of $F$ such that the following hold:

(1) We have $B B T_{T, p}(B)<\epsilon$.

(2) For every $b \in B$ we have $d_{T}(p, b p)<\epsilon$.

If $A$ is a free basis of a free group $F(A)$, for $u \in F(A)$ we denote by $|u|_{A}$ the freely reduced length of $u$ with respect to $A$ and we denote by $\|u\|_{A}$ the cyclically reduced length of $u$ with respect to $A$. Thus $\|u\|_{A}$ is the translation length of $u$ on the Cayley tree $X(F(A), A)$ of $F(A)$.

Proposition-Definition 4.5 (Double bounded cancellation constant). Let $A, B$ be two free bases of a finitely generated free group $F$. Then there exist integers $1 \leq l \leq n$ with the following properties: 
(1) Let $u, v$ be freely reduced words in $F(A)$ such that the word $u v$ is freely reduced. Let $u^{\prime}, v^{\prime}$ be freely reduced words in $F(B)$ representing $u, v$ accordingly. Then the maximal terminal segment of $u^{\prime}$ that freely cancels in the product $u^{\prime} \cdot v^{\prime}$ has length $\leq l$.

(2) If $u$ is a cyclically reduced word in $F(A)$ and $u^{\prime}$ is the freely reduced form of $u$ in $F(B)$ then $\left.\left|\left\|u^{\prime}\right\|_{B}-\right| u\right|_{B} \mid \leq 2 l$.

(3) Let $w=u w_{0} v$ be a freely reduced product of freely reduced words in $F(A)$ and suppose that $|u|_{A},|v|_{A}>n$. Let $x, y$ be freely reduced words in $F(A)$ such that the product $x w y$ is reduced as written. Let $x^{\prime}, w^{\prime}, y^{\prime}$ be the freely reduced forms of $x, w, y$ in $F(B)$. Let $w^{\prime \prime}$ be the maximal subword of $w^{\prime}$ that is not affected by the free cancellations in the product $x^{\prime} w^{\prime} y^{\prime}$. Then $w^{\prime \prime}$ is nonempty.

Let $z_{1}^{\prime}, z_{2}^{\prime}$ be freely reduced words in $F(B)$ such that $z_{1}^{\prime} w^{\prime \prime} z_{2}^{\prime \prime}$ is freely reduced as written. Let $z_{1}, z_{2}, \tilde{w}$ be the freely reduced words in $F(A)$ representing $z_{1}^{\prime}, z_{2}^{\prime}, w^{\prime \prime}$ accordingly. Then the maximal subword $W$ of $\tilde{w}$ that is not affected by the free cancellations in $z_{1} \tilde{w} z_{2}$ is nonempty and, in addition, $W$ contains $w_{0}$ as a subword. Moreover, if $z_{1}^{\prime} w^{\prime \prime} z_{2}^{\prime}$ is cyclically reduced in $F(B)$, then the maximal subword of $\tilde{w}$ that is not affected by the free and cyclic reduction of $z_{1} \tilde{w} z_{2}$, is nonempty and contains $w_{0}$ as a subword.

The existence of $l \geq 1$ as above follows from the bounded cancellation lemma [Coo]. It is not hard to deduce the existence of $n \geq 1$ from the existence of $l$.

We refer to the smallest integer $n=n_{A, B} \geq 1$ satisfying condition (3) above as the double-bounded cancellation constant for $A, B$.

Note that the definition of $n_{A, B}$ given above is not symmetric and it is possible that $n_{A, B} \neq n_{B, A}$. Note also that conditions (1),(2) in the above definition hold with $l=n_{A, B}$.

Definition 4.6. Let $A, B$ be two free bases of a finitely generated free group $F$.

Let $C=\max _{a \in A}|a|_{B}$. We call $C=C_{A, B}$ the Lipshitz constant for $A, B$.

\section{The "If" Case of the Main Result}

In this section we will establish the "if" implication in Theorem 1.1. Namely, we will prove that for an arbitrary very small action of $F$ on an $\mathbb{R}$-tree $T$ the condition $\operatorname{supp}(\mu) \subseteq L^{2}(T)$ implies that $\langle T, \mu\rangle=0$.

Remark 5.1. Suppose $\mu \in \operatorname{Curr}(F), \mu \neq 0$ and let

$$
\mu=\lim _{i \rightarrow \infty} \lambda_{i} \eta_{g_{i}}
$$

where $\lambda_{i} \geq 0$ and $g_{i} \in F$. Then for $\lambda_{i}^{\prime} \geq 0$ we have

$$
\mu=\lim _{i \rightarrow \infty} \lambda_{i}^{\prime} \eta_{g_{i}} \Longleftrightarrow \lim _{i \rightarrow \infty} \frac{\lambda_{i}^{\prime}}{\lambda_{i}}=1 .
$$

In particular (see $[\mathrm{Ka} 3,4]$ ), if $A$ is a free basis of $F$, and $\mu \in \operatorname{Curr}(F)$ is such that 
$\|\mu\|_{A}=1$ then for any $\lambda_{i} \geq 0, g_{i} \in F$, with $\mu=\lim _{i \rightarrow \infty} \lambda_{i} \eta_{g_{i}}$, we have

$$
\mu=\lim _{i \rightarrow \infty} \frac{1}{\left\|g_{i}\right\|_{A}} \eta_{g_{i}} .
$$

Theorem 5.2. Let $F$ be a finitely generated nonabelian free group. Let $T$ be a $\mathbb{R}$-tree with a very small minimal isometric action of $F$. Let $\mu \in \operatorname{Curr}(F)$ be such that $\operatorname{supp}(\mu) \subseteq L^{2}(T)$. Then $\langle T, \mu\rangle=0$.

Proof. Let $A$ be a free basis of $F$. We may assume that $\|\mu\|_{A}=1$. By Remark 5.1, it suffices to prove that whenever $w_{i}$ is a sequence of reduced cyclic words in $F(A)$ such that $\mu=\lim _{n \rightarrow \infty} \eta_{w_{i}} /\left\|w_{i}\right\|_{A}$ then $\lim _{i \rightarrow \infty}\left\|w_{i}\right\|_{T} /\left\|w_{i}\right\|_{A}=0$. Let $w_{i}$ be such a sequence of cyclic words. Note that $\left\|w_{i}^{t}\right\|_{T} /\left\|w_{i}^{t}\right\|_{A}=\left\|w_{i}\right\|_{T} /\left\|w_{i}\right\|_{A}$ and $\eta_{w_{i}^{t}} /\left\|w_{i}^{t}\right\|_{A}=$ $\eta_{w_{i}} /\left\|w_{i}\right\|_{A}$ for any integer $t \geq 1$. Thus, by taking powers if necessary, we may assume that $\lim _{i \rightarrow \infty}\left\|w_{i}\right\|_{A}=\infty$.

Let $p \in T$. Let $C_{1}>0$ be such that $B B T_{T, p}(A)<C_{1}$. Recall that such $C_{1}$ exists by Proposition 4.2. Also, we put $C_{2}=\max _{a \in A} d_{T}(p, a p)$.

Let $\epsilon>0$ be arbitrary. Let $N \geq 1$ be such that $\left(1+10 C_{1}\right) / N \leq \epsilon / 2$. Choose $\epsilon_{1}>0$ such that $C_{2} N \epsilon_{1} \leq \epsilon / 2$. Put $w_{i}^{\prime}=w_{i}^{N}$. Hence $\left\|w_{i}^{\prime}\right\|_{A}=N\left\|w_{i}\right\|_{A}$ is divisible by $N$ for every $i \geq 1$. Then $\eta_{w_{i}^{\prime}} /\left\|w_{i}^{\prime}\right\|_{A}=\eta_{w_{i}} /\left\|w_{i}\right\|_{A}$ and hence

$$
\mu=\lim _{i \rightarrow \infty} \frac{\eta_{w_{i}^{\prime}}}{\left\|w_{i}^{\prime}\right\|_{A}} .
$$

Write $w_{i}^{\prime}$ as a cyclic concatenation $u_{i}=y_{1} \ldots y_{m}$ where $m=\left\|w_{i}^{\prime}\right\|_{A} / N$ and where each $y_{j} \in F(A)$ has $\left|y_{j}\right|_{A}=N$. We say that $y_{j}$ is $\operatorname{good}$ if $y_{j} \in \operatorname{supp}_{A}(\mu)$ and that $y_{j}$ is bad otherwise. Write $m=m_{\text {good }}+m_{\text {bad }}$ where $m_{\text {good }}$ is the number of those $j=1, \ldots, m$ for which $y_{j}$ is good. Since $N$ is fixed and $\lim _{i \rightarrow \infty} \eta_{w_{i}^{\prime}} /\left\|w_{i}^{\prime}\right\|_{A}=\mu$, for any fixed freely reduced word $v \in F(A)$ of length $N$, the symmetrized frequencies $\left\langle v^{ \pm 1}, w_{i}^{\prime}\right\rangle_{A} /\left\|w_{i}^{\prime}\right\|_{A}$ of $v$ in $w_{i}^{\prime}$ converge to $\langle v, \mu\rangle_{A}$ as $i \rightarrow \infty$. In particular, if $v \notin \operatorname{supp}_{A}(\mu)$, these frequencies converge to zero. Therefore there exists some $i_{0} \geq 1$ such that for every $i \geq i_{0}$ we have $m_{b a d} \leq \epsilon_{1}\left\|w_{i}^{\prime}\right\|_{A}$. Suppose now that $i \geq i_{0}$ is arbitrary.

Let $z_{i} \in F(A)$ be the freely reduced word obtained as a (non-cyclic) concatenation $z_{i}=y_{1} \ldots y_{m}$.

Suppose first that $y_{j}$ is good. Since $\operatorname{supp}(\mu) \subseteq L^{2}(T)$, there exists a cyclically reduced word $u_{j} \in F(A)$ containing $y_{j}$ as an initial segment such that $\left\|u_{j}\right\|_{T} \leq 1$. Then by Lemma 4.3 we have $d_{T}\left(p, u_{j} p\right) \leq 1+2 C_{1}$. Write $u_{j}$ as a reduced product $u_{j}=y_{j} u_{j}^{\prime}$. Then Lemma 4.3 implies that

$$
d_{T}\left(p, y_{j} p\right)+d_{T}\left(p, u_{j}^{\prime} p\right) \leq d_{T}\left(p, u_{j} p\right)+2 C_{1} \leq 1+4 C_{1}
$$

and hence

$$
d_{T}\left(p, y_{j} p\right) \leq 1+4 C_{1} .
$$

Suppose that $y_{j}$ is bad. Then obviously $d_{T}\left(p, y_{j} p\right) \leq C_{2} N$.

Recall that $m_{\text {bad }} \leq \epsilon_{1}\left\|w_{i}^{\prime}\right\|_{A}$. Recall also that $z_{i}=y_{1} \ldots y_{m} \in F(A)$. Hence by Lemma 4.3

$$
\left\|w_{i}^{\prime}\right\|_{T}=\left\|z_{i}\right\|_{T} \leq d_{T}\left(p, z_{i} p\right) \leq m\left(1+4 C_{1}\right)+\epsilon_{1}\left\|w_{i}^{\prime}\right\|_{A} C_{2} N .
$$


Then for every $i \geq i_{0}$

and hence

$$
\frac{\left\|w_{i}^{\prime}\right\|_{T}}{\left\|w_{i}^{\prime}\right\|_{A}} \leq \frac{1+10 C_{1}}{N}+C_{2} N \epsilon_{1} \leq \epsilon,
$$

$$
0 \leq\langle T, \mu\rangle=\lim _{i \rightarrow \infty} \frac{\left\|w_{i}^{\prime}\right\|_{T}}{\left\|w_{i}^{\prime}\right\|_{A}} \leq \epsilon .
$$

Since $\epsilon>0$ was arbitrary, this implies that $\langle T, \mu\rangle=0$, as required.

\section{The Dense Orbits Case}

Proposition 6.1. Let $F$ be a finitely generated nonabelian free group. Let $T$ be an $\mathbb{R}$-tree with a very small minimal isometric action of $F$ such that this action has dense orbits.

Let $\mu \in \operatorname{Curr}(F)$ be such that $\langle T, \mu\rangle=0$. Then $\operatorname{supp}(\mu) \subseteq L^{2}(T)$.

Proof. Choose a free basis $A$ of $F$. By re-scaling we may assume that $\|\mu\|_{A}=1$. Then there exists a sequence of reduced cyclic words $w_{i}$ in $F(A)$ such that $\lim _{i \rightarrow \infty} \frac{\eta_{w_{i}}}{\left\|w_{i}\right\|}=\mu$. Since $\langle T, \mu\rangle=0$, we have $\lim _{i \rightarrow \infty} \frac{\left\|w_{i}\right\|_{T}}{\left\|w_{i}\right\|_{A}}=0$.

Again, recall that $\frac{\left\|w_{i}^{t}\right\|_{T}}{\left\|w_{i}^{t}\right\|_{A}}=\frac{\left\|w_{i}\right\|_{T}}{\left\|w_{i}\right\|_{A}}$ and $\frac{\eta_{w_{i}^{t}}}{\left\|w_{i}^{t}\right\|_{A}}=\frac{\eta_{w_{i}}}{\left\|w_{i}\right\|_{A}}$ for any integer $t \geq 1$. Thus, by taking powers if necessary, we may assume that $\lim _{i \rightarrow \infty}\left\|w_{i}\right\|_{A}=\infty$.

Let $v \in F(A)$ be a freely reduced word with $\langle v, \mu\rangle_{A}=\alpha>0$. We need to prove that for any $\epsilon>0$ there exists a cyclically reduced word $w$ in $F(A)$ containing $v$ as a subword and such that $\|w\|_{T} \leq \epsilon$.

Since $\|\mu\|_{A}=1$, there exists a sequence of cyclic words $w_{i}$ in $F(A)$ such that

$$
\lim _{i \rightarrow \infty} \frac{\eta_{w_{i}}}{\left\|w_{i}\right\|_{A}}=\mu
$$

and such that

$$
\lim _{i \rightarrow \infty} \frac{\left\|w_{i}\right\|_{T}}{\left\|w_{i}\right\|_{A}}=0
$$

Hence

$$
\lim _{i \rightarrow \infty} \frac{\left\langle v^{ \pm 1}, w_{i}\right\rangle}{\left\|w_{i}\right\|}=\langle v, \mu\rangle_{A}=\alpha>0 .
$$

Choose $i_{0} \geq 1$ such that for every $i \geq i_{0}$ we have

$$
\frac{\alpha}{2} \leq \frac{\left\langle v^{ \pm 1}, w_{i}\right\rangle}{\left\|w_{i}\right\|} \leq 2 \alpha .
$$

Let $\epsilon>0$ be arbitrary. By Proposition 4.4 there is some free basis $B$ of $F$ and a point $p \in T$ such that $B B T_{T, p}(B)<\epsilon$ and $d_{T}(p, b p)<\epsilon$ for every $b \in B$. Let $n=n_{A, B} \geq 1$ be the double-bounded cancellation constant for $A, B$ and let $C=C_{A, B} \geq 1$ be the Lipshitz constant for $A, B$.

Let $i \geq i_{0}$. Choose a maximal collection $v_{1}, \ldots, v_{m}$ of occurrences of $v^{ \pm 1}$ in $w_{i}$ such that the $n$-neighborhoods of these occurrences in $w_{i}$ do not overlap. Note that if an occurrence of $v^{ \pm 1}$ begins outside the union of the $n$-neighborhoods of $v_{1}, \ldots, v_{m}$ in $w_{i}$, then, by maximality, the beginning of that occurrence is at most $2 n+|v|$ 
away from the union of the $n$-neighborhoods of $v_{1}, \ldots, v_{m}$. This implies that the total number of occurrences of $v^{ \pm 1}$ in $w_{i}$ satisfies

$$
\left\langle v^{ \pm 1}, w_{i}\right\rangle \leq 2 m(2 n+|v|) .
$$

Hence

$$
\frac{m}{\left\|w_{i}\right\|_{A}} \geq \frac{\left\langle v^{ \pm 1}, w_{i}\right\rangle}{\left\|w_{i}\right\|_{A}(4 n+2|v|)} \geq \frac{\alpha}{2} \frac{1}{4 n+2|v|} .
$$

Thus,

$$
\frac{\alpha}{K} \leq \frac{m}{\left\|w_{i}\right\|} \leq 2 \alpha
$$

where $K=8 n+4|v|$.

Recall that $\lim _{i \rightarrow \infty} \frac{\left\|w_{i}\right\|_{T}}{\left\|w_{i}\right\|_{A}}=0$. Let $\epsilon_{1}>0$ be such that $\epsilon_{1} \frac{K}{\alpha}<\epsilon$. We may assume that $i \geq i_{0}$ was chosen big enough so that $\frac{\left\|w_{i}\right\|_{T}}{\left\|w_{i}\right\|_{A}} \leq \epsilon_{1}$.

We now subdivide the cyclic word $w_{i}$ as a cyclic concatenation $w=y_{1} \ldots y_{m}$ where each segment $y_{j} \in F(A)$ of $w_{i}$ contains the $n$-neighborhood of $v_{j}$ in $w_{i}$ for $j=$ $1, \ldots, m$. For $j=1, \ldots, m$ let $y_{j}^{\prime}$ be the freely reduced word in $F(B)$ representing $y_{j}$.

Let $z_{j}$ be the portion of $y_{j}^{\prime}$ that survives after the free reductions in $y_{j-1}^{\prime} y_{j}^{\prime} y_{j+1}^{\prime}$. Note that $z_{j}$ is nonempty by definition of $n=n_{A, B}$. Then the cyclic concatenation $w_{i}^{\prime}=y_{1}^{\prime} \ldots y_{m}^{\prime}$ is the cyclic word in $F(B)$ representing the same conjugacy class as $w_{i}$. Note that the words $y_{j}^{\prime}$ need not be cyclically reduced. We choose letters $b_{j} \in B^{ \pm 1}$ such that each $x_{j}:=b_{j} z_{j}$ is cyclically reduced over $B$ and such that $x_{1} \ldots x_{m}$ is a freely reduced and cyclically reduced word over $B$. Let $w_{i}^{\prime \prime}$ be the cyclic word obtained by cyclic concatenation $w_{i}^{\prime \prime}=x_{1} \ldots x_{m}$.

By Lemma 4.3 we have

$$
\begin{gathered}
\left|d_{T}\left(p, y_{1}^{\prime} \ldots y_{m}^{\prime} p\right)-\sum_{j=1}^{m} d_{T}\left(p, z_{j} p\right)\right| \leq 4 m \epsilon, \\
\left|d\left(p, x_{j} p\right)-d\left(p, z_{j} p\right)\right| \leq \epsilon, \text { and } \\
\left|d_{T}\left(p, x_{1} \ldots x_{m} p\right)-\sum_{j=1}^{m} d_{T}\left(p, z_{j} p\right)\right| \leq 4 m \epsilon .
\end{gathered}
$$

Hence

$$
\left|d_{T}\left(p, y_{1}^{\prime} \ldots y_{m}^{\prime} p\right)-d_{T}\left(p, x_{1} \ldots x_{m} p\right)\right| \leq 12 m \epsilon .
$$

Since both $y_{1}^{\prime} \ldots y_{m}^{\prime}$ and $x_{1} \ldots x_{m}$ are cyclically reduced, Lemma 4.3 also implies

$$
\begin{gathered}
\left|d_{T}\left(p, y_{1}^{\prime} \ldots y_{m}^{\prime} p\right)-\left\|w_{i}^{\prime}\right\|_{T}\right| \leq 2 \epsilon, \\
\left|d_{T}\left(p, x_{1} \ldots x_{m} p\right)-\left\|w_{i}^{\prime \prime}\right\|_{T}\right| \leq 2 \epsilon, \text { and hence } \\
\left|\left\|w_{i}^{\prime}\right\|_{T}-\left\|w_{i}^{\prime \prime}\right\|_{T}\right| \leq 12 m \epsilon+4 \epsilon .
\end{gathered}
$$

Thus

$$
\left\|w_{i}^{\prime \prime}\right\|_{T} \leq\left\|w_{i}^{\prime}\right\|_{T}+12 m \epsilon+4 \epsilon .
$$

Since $x_{1}, \ldots, x_{m}$ are cyclically reduced, Lemma 4.3 again implies that

$$
\sum_{j=1}^{m}\left\|x_{j}\right\|_{T} \leq\left\|w_{i}^{\prime \prime}\right\|_{T}+4 m \epsilon \leq\left\|w_{i}^{\prime}\right\|_{T}+16 m \epsilon+4 \epsilon
$$


Therefore there exists $j$ such that

$$
\left\|x_{j}\right\|_{T} \leq \frac{\left\|w_{i}^{\prime}\right\|_{T}}{m}+16 \epsilon+\frac{4 \epsilon}{m} \leq \frac{\left\|w_{i}^{\prime}\right\|_{T}}{m}+20 \epsilon .
$$

Recall that $w_{i}$ and $w_{i}^{\prime}$ represent the same conjugacy class in $F$ and so $\left\|w_{i}\right\|_{T}=\left\|w_{i}^{\prime}\right\|_{T}$. Thus

$$
\left\|x_{j}\right\|_{T} \leq \frac{\left\|w_{i}\right\|_{T}}{m}+20 \epsilon=\frac{\left\|w_{i}\right\|_{T}}{\left\|w_{i}\right\|_{A}} \frac{\left\|w_{i}\right\|_{A}}{m}+20 \epsilon \leq \epsilon_{1} \frac{K}{\alpha}+20 \epsilon \leq 21 \epsilon
$$

where the last inequality holds by the choice of $\epsilon_{1}$.

We now rewrite $x_{j}=b_{j} z_{j}$ as a freely reduced word in $F(A)$ and then cyclically reduce the result to get a cyclically reduced word $s_{j}$ in $F(A)$. By definition of $n=n_{A, B}$, the construction of $x_{j}$ implies that the occurrence $v_{j}$ of $v^{ \pm 1}$ in $y_{j}$ survives intact in $s_{j}$. Thus $s_{j}$ is a cyclically reduced word in $F(A)$ containing $v^{ \pm 1}$ as a subword and satisfying $\left\|s_{j}\right\|_{T} \leq 21 \epsilon$. Since $\epsilon>0$ was arbitrary, this implies that $v \in L^{2}(T)$, as required.

\section{Approximating the Word Metric for a Graph of Groups}

Convention 7.1. If $G=\langle S\rangle$ is a group with a finite-generating set $S$, we say that a cyclic word $w$ in $S^{ \pm 1}$ is a cyclic $\lambda$-quasigeodesic with respect to $d_{S}$ if for every vertex on $w$ cutting $w$ open at that vertex produced a word that is $\lambda$-quasigeodesic. We will denote the Cayley graph of $X$ with respect to $S$ by $X(G, S)$.

Lemma 7.2. Let $G$ be a word-hyperbolic group with a fixed finite generating set $S$ and let $\lambda>0$. Then there exists $\lambda^{\prime}=\lambda^{\prime}(\lambda, S, G)>0$ with the following property. Suppose that $w$ is a cyclic word in $S^{ \pm 1}$ that is a cyclic $\lambda$-quasigeodesic for $G$ with respect to $d_{S}$. Let $w$ be subdivided as a cyclic concatenation $w=v_{1} \ldots v_{m}$ where each $v_{i}$ is a $\lambda$-quasigeodesic word in $S^{ \pm 1}$. For $i=1, \ldots, m$ let $z_{i}$ be a another $\lambda$-quasigeodesic word representing the same element of $G$ as $v_{i}$. Let $w^{\prime}$ be the cyclic word obtained by a cyclic concatenation $w^{\prime}=z_{1} \ldots z_{m}$. Then $w^{\prime}$ is a cyclic $\lambda^{\prime}$-quasigeodesic.

Proof. The proof is a straightforward variation on the proof of a similar Lemma 3.4 in [Ka1] for a non-cyclic word and we leave the details to the reader. Note, however, that the proof uses the fact that $G$ is word-hyperbolic and hence the paths labelled by $z_{i}$ and $v_{i}$ in the Cayley graph of $G$ are $\epsilon$-Hausdorff close, where $\epsilon=\epsilon(\lambda, G, S)>0$. It is not hard to see that the statement of this lemma in general fails for non-hyperbolic groups, e.g. for $\mathbb{Z}^{2}$.

Notation 7.3 (Graph of groups notation). Let $\mathcal{Y}$ be a graph of groups [B], [S]. For $x \in V Y$ we will denote the vertex group of $x$ by $G_{x}$ and for $e \in E Y$ we will denote the edge group of $e$ by $G_{e}$. For $e \in E Y$ we denote the initial vertex of $e$ by $o(e)$ and we denote the terminal vertex of $e$ by $t(e)$. For $e \in E Y$ we denote the corresponding boundary monomorphisms by $\alpha_{e}: G_{e} \rightarrow G_{o(e)}$ and $\omega_{e}: G_{e} \rightarrow G_{o(e)}$. Recall that according to the standard graph of groups conventions, for every $e \in E Y$ we have $G_{e}=G_{e^{-1}}, o(e)=t\left(e^{-1}\right)$ and $\alpha_{e}=\omega_{e^{-1}}$. 
Definition 7.4. Let $\mathcal{Y}$ is a finite connected reduced graph of groups with underlying graph $Y$, where all the vertex groups $G_{x}, x \in V Y$, are finitely generated. Let $Z \subseteq Y$ be a maximal tree in $Y$. Fix an orientation $E Y=E^{+} Y \sqcup E^{-} Y$ on $Y$, so that for every $e \in E Y$ we have $e \in E^{+} Y$ iff $e^{-1} \in E^{-} Y$.

Let $G=\pi_{1}(\mathcal{Y}, Z)$. Then $G$ has the presentation

$$
\begin{aligned}
& G=\left(\left(\star_{x \in V Y} G_{x}\right) *\right.\left.F\left(E^{+} Y\right)\right) / \\
&\left\langle\left\langle e^{-1} \alpha_{e}(c) e=\omega_{e}(c), e \in E^{+} Y, c \in G_{e} ; e=1, e \in E^{+} Z\right\rangle\right\rangle .
\end{aligned}
$$

Let $S_{x}$ be a finite generating set of $G_{x}$ for $x \in V Y$. Then

$$
S=S y=E^{+} Y \bigcup \cup_{x \in V Y} S_{x}
$$

is a generating set of $G$ that is said to be adapted to $\mathcal{Y}$.

Recall that a $\mathcal{Y}$-path from $x \in V Y$ to $x^{\prime} \in V Y$ is a sequence

$$
\alpha=g_{0}, e_{1}, g_{1}, e_{2}, \ldots, e_{n}, g_{n}
$$

where $e_{1}, \ldots, e_{n}$ is an edge-path in $Y$ with the vertex sequence $x=x_{0}, x_{1}, \ldots, x_{n}=x^{\prime}$ and where $g_{i} \in G_{x_{i}}$. A $\mathcal{Y}$-path $\alpha$ as above is $\mathcal{Y}$-reduced if it does not contain a subsequence of the form

$$
e, \omega_{e}(c), e^{-1},
$$

where $e \in E Y$ and $c \in G_{e}$.

We say that a word $W$ in $S_{\mathcal{Y}}^{ \pm 1}$ is a path-word for $\mathcal{Y}$ if it has the form

$$
W=w_{0} e_{1} w_{1} \ldots e_{n} w_{n}
$$

where $e_{1}, \ldots, e_{n}$ is an edge-path in $Y$ with the vertex sequence $x_{0}, x_{1}, \ldots, x_{n}$ and where $w_{i}$ is a word in $S_{x_{i}}^{ \pm 1}$. Note that any path-word $W$ as above defines a $\mathcal{Y}$-path $\alpha=g_{0}, e_{1}, g_{1}, e_{2}, \ldots, e_{n}, g_{n}$, where $g_{i} \in G_{x_{i}}$ is the group element represented by $w_{i}$.

We say that a path-word $W$ as above is a $\mathcal{Y}$-reduced path word if it defines a $\mathcal{Y}$-reduced $\mathcal{Y}$-path.

In a similar way, one defines the notions of a cyclic $\mathcal{Y}$-path, $\mathcal{Y}$-reduced cyclic $\mathcal{Y}$-path, a cyclic path-word for $\mathcal{Y}$ and a $\mathcal{Y}$-reduced cyclic path-word for $\mathcal{Y}$.

Convention 7.5. Let $A$ be another finite generating set for $G=\pi_{1}(\mathcal{Y}, Z)$. For each element of $S y$ choose its representation as a word in $A$. Using these representations for substitution, any (cyclic) word $W$ in $S_{\mathcal{Y}}^{ \pm 1}$ defines a (cyclic) word $\widetilde{W}$ in $A^{ \pm 1}$.

Proposition 7.6. Let $G$ be a word-hyperbolic group and let $G=\pi_{1}(\mathcal{Y}, Z)$ be a splitting of $G$ where $\mathcal{Y}$ is a finite graph of groups, and where $Z \subseteq Y$ is a maximal subtree. Suppose that each vertex group $G_{x}$ is quasiconvex in $G$. Let $S_{x}$ be a finite generating set of $G_{x}$ for each $x \in V Y$ and let

$$
S_{y}=E^{+} Y \bigcup \cup_{x \in V Y} S_{x}
$$

be a generating set of $G$ adapted to $\mathcal{Y}$. Let $A$ be any other finite generating set of $G$.

There exist $\lambda>0$ and $\epsilon>0$ with the following properties: 
(1) For any $g \in F, g \neq 1$ there exists a $\mathcal{Y}$-reduced path-word $W$ representing $g$ such that $W$ is $\lambda$-quasigeodesic with respect to $d_{S y}$.

Moreover, if $w \in\left(A \cup A^{-1}\right)^{*}$ is a $d_{A}$-geodesic representing $g$, then the paths from 1 to $g$ labelled by $w$ and $\tilde{W}$ in $X(F, A)$ are $\epsilon$-Hausdorff close.

(2) For any conjugacy class $[g], g \in F, g \neq 1$, there exists a $\mathcal{Y}$-reduced cyclic path-word representing $[g]$ such that $W$ is a cyclic $\lambda$-quasigeodesic with respect to $d_{S y}$.

Moreover, let $w$ be a cyclic word over $A^{ \pm 1}$ which is a cyclic $d_{A}$-geodesic representing $[g]$. Then there is a function $f$ from the vertex set of $w$ to the vertex set of $W$ with the following properties. For every vertex $x$ on $w$ cutting open $w$ at $x$ and $W$ at $f(x)$ produces a $d_{A}$-geodesic word $w_{x}$ and a $\mathcal{Y}$-reduced path-word $W_{x}$ accordingly. Additionally, there exist paths $p$ and $p^{\prime}$ in $X(G, A)$ labelled by $w_{x}$ and $\widetilde{W}_{x}$ such that there initial vertices are at most $\epsilon$-apart, their terminal vertices are at most $\epsilon$-apart and, for any other vertex $y$ on $w$ the vertices on $p$ and $p^{\prime}$ corresponding to $y$ and $f(y)$ are at most $\epsilon$-apart in $X(G, A)$.

Proof. Part (1) of the lemma is essentially the same as Proposition 5.1 in [Ka2]. We will briefly indicate the proof of part (2) that is similar to the proof of Proposition 5.1 in $[\mathrm{Ka} 2]$.

To see that part (2) holds, we first replace $g$ by a $d_{S \mathcal{Y}}$-shortest element $g^{\prime} \in[g]$. Let $\hat{W}$ be a $d_{S_{\mathcal{Y}}}$-geodesic representative of $g^{\prime}$. Then, by the choice of $g^{\prime}$, every cyclic

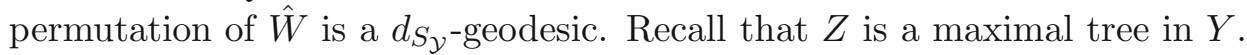

Let $W_{0}$ be the cyclic word defined by $\hat{W}$. Thus $W_{0}$ is a cyclic $d_{S_{\mathcal{Y}}}$-geodesic. By inserting in $W_{0}$ several subwords in the alphabet $E Z$ (which therefore represent the trivial element of $G$ ) of length $\leq \# E Z$ each, we can obtain a new cyclic word $W_{1}$ that is a cyclic path-word for $\mathcal{Y}$. By Lemma $7.2 W_{1}$ is a cyclic $\lambda_{1}$-quasigeodesic for some constant $\lambda_{1}>0$. However, $W_{1}$ need not be $\mathcal{Y}$-reduced.

We say that a subsegment $v$ of $W_{1}$ is a pinch if $v$ has the form $v=e u e^{-1}$ and $v$ represents an element of the edge group $\omega_{e}\left(G_{e}\right)$. Note that we DO NOT require $u$ to be a word in the generating set of the vertex group $G_{t(e)}$. We say that a collection of pinches $v_{1}, \ldots, v_{m}$ in $W_{1}$ is separated if the segments $v_{1}, \ldots, v_{m}$ do not overlap in $W_{1}$.

Let each of $v_{1}, \ldots, v_{m}$ and $v_{1}^{\prime}, \ldots, v_{l}^{\prime}$ be a separated collection of pinches in $W_{1}$. We say that $v_{1}, \ldots, v_{m}$ is dominated by $v_{1}^{\prime}, \ldots, v_{l}^{\prime}$ if for each $v_{i}$ there is some $v_{j}^{\prime}$ such that the segment $v_{i}$ is contained in the segment $v_{j}$ in $W_{1}$.

Now take $v_{1}=e_{1} u_{1} e_{1}^{-1}, \ldots, v_{m}=e_{m} u_{m} e_{m}^{-1}$ be a maximal (with respect to dominance) separated collection of pinches in $W_{1}$. Replace each $v_{i}$ in $W_{1}$ by its geodesic representative in the generators of the vertex group $G_{o\left(e_{i}\right)}$. Denote the resulting cyclic word by $W$.

Since the vertex groups are quasiconvex in $G$, Lemma 7.2 implies that the cyclic word $w$ is a cyclic $\lambda_{2}$-quasigeodesic for some constant $\lambda_{2}>0$. The maximality of the choice of $v_{1}, \ldots, v_{m}$ implies that $W$ is a $\mathcal{Y}$-reduced cyclic path-word. 
Establishing the remaining properties of $W$ asserted in part (2) of the proposition is a straightforward $\delta$-hyperbolic exercise and we leave the details to the reader.

\section{The Discrete Action Case}

Convention 8.1. For the remainder of this section, let $F$ be a finitely generated nonabelian free group. We fix a very small splitting $F=\pi_{1}(\mathcal{Y}, Z)$, where $\mathcal{Y}$ is a finite connected reduced graph of groups with a maximal tree $Z$, where all edge groups are cyclic (and hence all vertex groups are finitely generated free groups). We fix an orientation on $Y$ and choose a finite generating set

$$
S_{\mathcal{Y}}=E^{+} Y \bigcup \cup_{x \in V Y} S_{x}
$$

for $F$ that is adapted to $\mathcal{Y}$.

Let $V^{\prime} Y$ denote the set of all $x \in V Y$ such that the vertex group $G_{x}$ is non-cyclic. Let $T_{\mathcal{Y}}$ be the Bass-Serre tree corresponding to the splitting $F=\pi_{1}(\mathcal{Y}, Z)$.

We also fix a free basis $A$ for $F$.

Lemma 8.2. Let $L=L^{2}\left(T_{\mathcal{Y}}\right)$ and let $v \in F(A)$ be freely reduced. Then $v \in L_{A}$ if and only if for some vertex $x \in V^{\prime} Y$ the word $v$ can be read as the label of a path in the core of the Stallings subgroup graph for $G_{x}$ with respect to $F(A)$. That is, $L=\cup_{x \in V^{\prime} Y} i_{\Lambda}\left(\partial^{2} G_{x}\right)$

Proof. Note first that, since $\mathcal{Y}$ defines a very small splitting of $F$, every cyclic vertex group of $\mathcal{Y}$ is contained in some non-cyclic vertex group. Hence every word in $F(A)$ readable in the core of the Stallings subgroup graph of a cyclic vertex group is also readable in the core of the Stallings subgroup graph of a non-cyclic vertex group.

Since the action of $F$ on $T y$ is discrete, there is some $c>0$ such that for every $f \in F$ either $\|f\|_{T_{\mathcal{Y}}} \geq c$ or else $f$ is conjugate to an element of some $G_{x}, x \in V Y$ and $\|f\|_{T_{\mathcal{Y}}}=0$. Therefore, in view of the above remark, by definition of $L=L^{2}\left(T_{\mathcal{Y}}\right)$, for $v \in F(A)$ we have $v \in L_{A}$ if and only if $v$ is a subword of some cyclically reduced word in $F(A)$ representing an element conjugate to an element of some $G_{x}, x \in V^{\prime} Y$. This implies the statement of the lemma.

The following lemma is an easy corollary of the definitions.

Lemma 8.3. Let $H \leq F(A)$ be a finitely generated subgroup. Let $M$ be the number of edges in the Stallings subgroup graph $\Gamma_{H}$ of $H$ with respect to $F(A)$. Let $z_{1}, w, z_{2}$ be freely reduced words in $F(A)$ such that $\left|z_{1}\right|,\left|z_{2}\right| \leq c$ and such that $z_{1} \cdot w \cdot z_{2} \in H$ (we do not assume this product to be freely reduced). Let $w$ be written as a reduced product $w=w_{1} w^{\prime} w_{2}$ where $\left|w_{1}\right|_{A},\left|w_{2}\right|_{A} \geq c+M$. Then $w^{\prime}$ can be read as the label of a path in the core of the graph $\Gamma_{H}$.

Proposition 8.4. Let $F$ be as in Convention 8.1. Let $T=T_{\mathcal{Y}}$ be the Bass-Serre tree corresponding to $\mathcal{Y}$. Let $\mu \in \operatorname{Curr}(F)$ be such that $\langle T, \mu\rangle=0$.

Then $\operatorname{supp}(\mu) \subseteq L^{2}(T)$.

Proof. Choose a free basis $A$ of $F$. By re-scaling we may assume that $\|\mu\|_{A}=1$. Let $w_{i}$ be a sequence of reduced cyclic words $w_{i}$ in $F(A)$ such that $\lim _{i \rightarrow \infty} \frac{\eta_{w_{i}}}{\left\|w_{i}\right\|}=\mu$. 
Since $\langle T, \mu\rangle=0$, we have $\lim _{i \rightarrow \infty} \frac{\left\|w_{i}\right\|_{T}}{\left\|w_{i}\right\|_{A}}=0$. As before, by taking powers if necessary, we may assume that $\lim _{i \rightarrow \infty}\left\|w_{i}\right\|_{A}=\infty$. We need to show that $\operatorname{supp}(\mu) \subseteq L^{2}(T)$.

Let $\lambda, \epsilon>0$ be as provided by Proposition 7.6. Let $M$ be the maximum of the numbers of edges among the Stallings subgroups graphs with respect to $F(A)$ for the subgroups $G_{x}, x \in V Y$.

Let $v \in F(A)$ be a freely reduced word such that $v \in \operatorname{supp}_{A}(\mu)$. Thus $\langle v, \mu\rangle_{A}>0$. Then there exists a freely reduced word $v^{\prime}=u_{1} v u_{2}$ such that $\alpha:=\left\langle v^{\prime}, \mu\right\rangle_{A}>0$ and such that $\left|u_{1}\right|_{A},\left|u_{2}\right|_{A} \geq M+\epsilon$. We may assume that for every $i \geq 1$ we have

$$
\alpha / 2 \leq \frac{\left\langle\left(v^{\prime}\right)^{ \pm 1}, w_{i}\right\rangle}{\left\|w_{i}\right\|_{A}} \leq 2 \alpha .
$$

Let $W_{i}$ be a cyclically $\lambda$-quasigeodesic $\mathcal{Y}$-reduced cyclic path-word representing the same conjugacy class in $F$ as $w_{i}$.

Fix $N \gg \epsilon$.

By the same argument as in the proof of Proposition 6.1 we may find $m$ distinct occurrences $v_{1}, \ldots, v_{m}$ of $\left(v^{\prime}\right)^{ \pm 1}$ in $w_{i}$ such that $N$-neighborhoods of $v_{1}, \ldots, v_{m}$ do not overlap in $w_{i}$ and such that

$$
\frac{\alpha}{K} \leq \frac{m}{\left\|w_{i}\right\|_{A}} \leq 2 \alpha
$$

where $K=8 N+4\left|v^{\prime}\right|$.

For each $j=1, \ldots, m$ let $x_{j}, x_{j}^{\prime}$ be the initial and terminal vertices of $v_{j}$ in $w_{i}$. Let $V_{j}$ be the segment of $W_{i}$ from $f\left(x_{j}\right)$ to $f\left(x_{j}^{\prime}\right)$. The fact that the occurrences $v_{1}, \ldots, v_{m}$ of $\left(v^{\prime}\right)^{ \pm 1}$ in $w$ have non-overlapping $N$-neighborhoods implies that the segments $V_{1}, \ldots, V_{m}$ in $W_{i}$ have no overlaps.

Suppose first that for every $w_{i}$ each $V_{j}$ contains an occurrence of some $e \in E Y$. Then $\left\|w_{i}\right\|_{T}=\left\|W_{i}\right\|_{T} \geq m$. Hence for every $i \geq 1$

$$
\frac{\left\|w_{i}\right\|_{T}}{\left\|w_{i}\right\|_{A}} \geq \frac{m}{\left\|w_{i}\right\|_{A}} \geq \frac{\alpha}{K}>0 .
$$

This contradicts the assumption that $\lim _{i \rightarrow \infty} \frac{\left\|w_{i}\right\|_{T}}{\left\|w_{i}\right\|_{A}}=0$. Thus there is some $w_{i}$ such that some $V_{j}$ contains no occurrences of $E Y$. Since $W_{i}$ is a cyclic path-word for $\mathcal{Y}$, this implies that there is some vertex $x$ of $Y$ such that $V_{j}$ is a word in $S_{x}^{ \pm 1}$.

Proposition 7.6 implies that in $X(F, A)$ there are paths $\alpha_{1}, \alpha_{2}$ labelled by $v^{\prime}$ and a word in $S_{x}^{ \pm 1}$ accordingly, such that the initial vertices of $\alpha_{1}, \alpha_{2}$ are at most $\epsilon$-apart and the terminal vertices of $\alpha_{1}, \alpha_{2}$ are at most $\epsilon$-apart. Recall that $v^{\prime}=u_{2} v u_{2}$ where $\left|u_{1}\right|,\left|u_{2}\right| \geq M+\epsilon$. Therefore by Lemma $8.3 v$ can be read as a path in the Stallings core graph of $G_{x}=\left\langle S_{x}\right\rangle \leq F(A)$. Hence by Lemma $8.2 v \in L^{2}(T)_{A}$, as required.

\section{Restricting Geodesic Currents to Subgroups}

DEFINITION 9.1 (Restriction of a current to a subgroup). Let $H \leq F$ be a finitely generated nonabelian subgroup. Since $H$ is quasiconvex in $F$, we have a canonical $H$-equivariant topological embedding $\partial H \subset \partial F$ which induces a canonical $H$ equivariant topological embedding $\partial^{2} H \subset \partial^{2} F$. Let $\mu \in \operatorname{Curr}(F)$. We define the 
restricted current $\left.\mu\right|_{H}$ as follows. For any Borel subset $S \subseteq \partial^{2} H$ put $\left.\mu\right|_{H}(S):=\mu(S)$. It is easy to see that $\left.\mu\right|_{H}$ is an $H$-invariant measure on $\partial^{2} H$, that is $\left.\mu\right|_{H} \in \operatorname{Curr}(H)$.

Denote $r_{H}: \operatorname{Curr}(F) \rightarrow \operatorname{Curr}(H), r_{H}(\mu)=\left.\mu\right|_{H}$ for $\mu \in \operatorname{Curr}(F)$.

REMARK 9.2. In the above definition, the restricted current $\left.\mu\right|_{H}$ depends only on $\mu$ and the conjugacy class of $H$ in $F$ in the following sense. Let $f \in F$ and put $H_{1}=f H f^{-1}$. Consider an isomorphism $\alpha: H \rightarrow H_{1}$ defined as $\alpha(h)=f h f^{-1}$ for $h \in H$. Then $\alpha$ induces an $\alpha$-equivariant homeomorphism $\widehat{\alpha}: \partial^{2} H \rightarrow \partial^{2} H_{1}$. Hence $\widehat{\alpha}$ induces a canonical isomorphism $\breve{\alpha}: \operatorname{Curr}(H) \rightarrow \operatorname{Curr}\left(H_{1}\right)$ defined as $(\breve{\alpha} \nu)\left(S_{1}\right)=\nu\left(\widehat{\alpha}^{-1}\left(S_{1}\right)\right)$ for any $\nu \in \operatorname{Curr}(H)$ and $S_{1} \subseteq \partial^{2} H_{1}$.

It is not hard to check that for any $\mu \in \operatorname{Curr}(F)$ we have $\breve{\alpha}\left(\left.\mu\right|_{H}\right)=\left.\mu\right|_{H_{1}}$.

The following is an immediate corollary of the definitions.

Proposition 9.3. Let $H \leq F$ be a finitely generated nonabelian subgroup. Then $r_{H}: \operatorname{Curr}(F) \rightarrow \operatorname{Curr}(H)$ is a linear continuous map.

Notation 9.4. Let $G$ be a group and $g \in G$ be an element. We denote by $[g]_{G}$ the conjugacy class of $g$ in $G$.

The proof of the following lemma given below was suggested to the authors by Gilbert Levitt.

Lemma 9.5. Let $H \leq F$ be a finitely generated subgroup.

(1) There exists an integer $n \geq 1$ with the following property. For every $h \in H$ there exist $h_{1}, \ldots, h_{m} \in H$ with $m \leq n$ such that

$$
[h]_{F} \cap H=\left[h_{1}\right]_{H} \cup\left[h_{2}\right]_{H} \cup \cdots \cup\left[h_{m}\right]_{H} .
$$

We denote by $n_{H, F}$ the smallest $n \geq 1$ with this property.

(2) There exists an integer $n_{1} \geq 1$ with the following property. Let $h \in H$ be any nontrivial element that is not a proper power in $H$. Represent $h$ as $h=f^{d}$ where $f \in F$ is not a power and $d \geq 1$. Then $d \leq n_{1}$.

We denote by $d_{H, F}$ the smallest $n_{1} \geq 1$ with this property.

(3) A finitely generated subgroup $H \leq F$ is malnormal if and only if $n_{H, F}=$ $d_{H, F}=1$.

Proof. It is easy to see that if $H \leq F$ is malnormal then $n_{H, F}=d_{H, F}=1$ satisfy the requirements of the lemma. Similarly, if $n_{H, F}=d_{H, F}=1$, it is not hard to show that $H \leq F$ is malnormal. Thus part (3) of the lemma holds.

Suppose now that $H \leq F$ is an arbitrary finitely generated subgroup. By Marshall Hall's theorem there exist subgroups $K, U \leq F$ such that $U=\langle H, K\rangle=H * K$ has finite index in $F$. Let $p=[F: U]$. Choose $f_{1}, \ldots, f_{p} \in F$ such that $F=$ $\cup_{j=1}^{p} U f_{j}$.

Let $h \in H, h \neq 1$ be arbitrary. Let $J=\left\{j \mid 1 \leq j \leq p, f_{j}^{-1} h f_{j} \in U\right\}$. Every element of $F$ has the form $u f_{j}$, where $u \in U, 1 \leq j \leq p$. Hence it is obvious that

$$
[h]_{F} \cap U=\cup_{j \in J}\left[f_{j}^{-1} h f_{j}\right]_{U}
$$

Let $J^{\prime}=\left\{j \in J: f_{j}^{-1} h f_{j} \in H\right\}$. Since $H \leq U=H * K$ is malnormal in $U$, it follows that for $j \in J^{\prime}$ we have

$$
\left[f_{j}^{-1} h f_{j}\right]_{U} \cap H=\left[f_{j}^{-1} h f_{j}\right]_{H}
$$


and for $j \in J-J^{\prime}$ we have

$$
\left[f_{j}^{-1} h f_{j}\right]_{U} \cap H=\varnothing
$$

Therefore

$$
[h]_{F} \cap H=\cup_{j \in J^{\prime}}\left[f_{j}^{-1} h f_{j}\right]_{H} .
$$

Since $h \in H, h \neq 1$, was arbitrary, we see that $n_{H, F} \leq p=[F: U]$ and part (1) of the lemma is proved.

Now let $U_{1} \leq U$ be such that $U_{1} \leq F$ is normal and of finite index. Let $q=\left[F: U_{1}\right]$. It is obvious that for any $f \in F$ we have $f^{q} \in U_{1}$ and thus $f^{q} \in U$.

Now let $h \in H, h \neq 1$, be an element that is not a proper power in $H$. Let $h=f^{d}$ where $f \in F, d \geq 1$, and where $f \in F$ is not a proper power. Note that since $H \leq U=U * K$ is malnormal in $U$, it follows that $h$ is not a proper power in $U$. Let $t \geq 1$ be the smallest such that $f^{t} \in U$. Note that, by the above remark, $t \leq q=\left[F: U_{1}\right]$. Note also, that, since $H$ is a free factor of $U$ and since $\left(f^{t}\right)^{d}=h^{t} \in H$, it follows that $f^{t} \in H$.

Suppose that $d>t$. If $d$ is divisible by $t, d=t s$, then $f^{t} \in U$ and $f^{t s} \in H$, where $s>1$. Since $f^{t} \in H$ and $h=\left(f^{t}\right)^{s}$, this contradicts our assumption that $h \in H$ is not a proper power in $H$. If $d$ is not divisible by $t$, we can write $d=t s+r$ where $0<r<t$. Since $f^{d}=h \in U, f^{t} \in U$, it follows that $f^{r} \in U$. Since $0<r<t$, this contradicts the choice of $t$. Thus $d \leq t \leq q=\left[F: U_{1}\right]$. Hence $d_{H, F} \leq\left[F: U_{1}\right]$ and part (2) of the lemma is proved.

The next lemma follows easily from the definitions of counting currents in terms of delta-functions.

Lemma 9.6. Let $H \leq F$ be a nonabelian finitely generated subgroup. Let $h \in H$ be a nontrivial element such that $h$ is not a proper power in $H$. Let $h_{1}, \ldots, h_{m} \in H$ be pairwise non-conjugate elements such that $[h]_{F} \cap H=\left[h_{1}\right]_{H} \cup\left[h_{2}\right]_{H} \cup \cdots \cup\left[h_{m}\right]_{H}$. Let $h=f^{d}$ where $d \geq 1$ and $f \in F$ is not a proper power in $F$.

Then,

(1) We have $\left.\eta_{h}^{F}\right|_{H}=d \sum_{i=1}^{m} \eta_{h_{i}}^{H}$.

(2) If $H \leq F$ is malnormal then $d=m=1$ and $\left.\eta_{h}^{F}\right|_{H}=\eta_{h}^{H}$.

Lemma 9.7. Let $H \leq F$ be a finitely generated subgroup. Let $A$ be a free basis of $F$ and let $\Gamma_{H}$ be the Stallings subgroup graph for $H \leq F$ with respect to $A$.

Let $\mu \in \operatorname{Curr}(F)$. Then $\operatorname{supp}(\mu) \subseteq i_{\Lambda}\left(\partial^{2} H\right)$ if and only if for every $v \in F(A)$ with $\langle v, \mu\rangle>0$ there is a reduced edge-path labelled by $v$ in $\operatorname{Core}\left(\Gamma_{H}\right)$. That is, $\operatorname{supp}(\mu) \subseteq i_{\Lambda}\left(\partial^{2} H\right)$ if and only if for each $v \in F(A)$ that cannot be read along a reduced path in $\operatorname{Core}\left(\Gamma_{H}\right)$ we have $\langle v, \mu\rangle=0$.

Proof. Note that the convex hull $T$ of $\partial H$ in $X(F, A)$ is a minimal $H$-invariant subtree in $X(F, A)$ and that $T$ can also be seen as a copy of the universal cover of $\operatorname{Core}\left(\Gamma_{H}\right)$ in $X(F, A)$.

It is not hard to see that for $v \in F(A)$ exactly one of the following two alternatives holds:

(a) Every segment labelled by $v$ in $X(F, A)$ is contained in some $F$-translate of $T$ in $F(X, A)$ and every such segment defines a two-sided cylinder that intersects nontrivially some $F$-translate of $\partial^{2} H$ in $\partial^{2} F$. 
(b) No segment labelled by $v$ in $X(F, A)$ is contained in an $F$-translate of $T$ and every such segment defines a two-sided cylinder that is disjoint from every $F$-translate of $\partial^{2} H$ in $\partial^{2} F$.

This fact immediately implies the statement of the lemma.

Notation 9.8. Let $H \leq F$ be a finitely generated subgroup. We denote by $\operatorname{Curr}_{H}(F)$ the set of all $\mu \in \operatorname{Curr}(F)$ such that $\operatorname{supp}(\mu) \subseteq i_{\Lambda}\left(\partial^{2} H\right)$.

Lemma 9.7 immediately implies

Lemma 9.9. Let $H \leq F$ be a finitely generated subgroup. Then $\operatorname{Curr}_{H}(F)$ is a closed affine subspace of $\operatorname{Curr}(F)$.

Proposition 9.10. Let $H \leq F$ be a finitely generated subgroup and let $\mu \in$ $\operatorname{Curr}_{H}(F)$. Then there exist a sequence $h_{i} \in H$ and a sequence $\lambda_{i} \geq 0$ such that

$$
\mu=\lim _{i \rightarrow \infty} \lambda_{i} \eta_{h_{i}}^{F} \text {. }
$$

Proof. Fix a free basis $A$ of $F$. By re-scaling we may assume that $\|\mu\|_{A}=1$. Let $w_{i}$ be a sequence of reduced cyclic words in $F(A)$ such that $\lim _{i \rightarrow \infty} \frac{\eta_{w_{i}}}{\left\|w_{i}\right\|_{A}}=\mu$.

Let $\Gamma_{H}$ be the Stallings subgroup graph of $H$ with respect to $A$. Let $C>0$ be such that for any directed edges $e, e^{\prime}$ in $\operatorname{Core}\left(\Gamma_{H}\right)$ there exists an edge-path $p$ of length $\leq C$ in $\operatorname{Core}\left(\Gamma_{H}\right)$ such that $e p e^{\prime}$ is a reduced edge-path in $\operatorname{Core}\left(\Gamma_{H}\right)$.

It suffices to prove that for every $M \geq 1$ and every $\epsilon>0$ there exists a cyclic path in $\operatorname{Core}\left(\Gamma_{H}\right)$ labelled by a cyclic word $w$ such that for every $v \in F(A)$ with $|v|_{A}=M$ we have

$$
\left|\frac{\left\langle v^{ \pm 1}, w\right\rangle}{\|w\|_{A}}-\langle v, \mu\rangle\right| \leq \epsilon .
$$

Let an integer $M \geq 1$ and a real number $\epsilon>0$ be arbitrary. Let $\alpha=$ $\max _{|v|_{A}=M}\langle v, \mu\rangle$.

Choose an integer $N>M \geq 1$ be such that

$$
(\alpha+\epsilon)\left(1-\frac{N}{N+C}\right) \leq \epsilon
$$

and such that $\frac{M+C}{N} \leq \frac{\epsilon}{3}$.

By replacing $w_{i}$ by their high powers if necessary, we may assume that $\left\|w_{i}\right\|_{A}$ is divisible by $N$ for every $i \geq 1$ and that $\lim _{i \rightarrow \infty}\left\|w_{i}\right\|_{A}=\infty$.

Since $\mu=\lim _{i \rightarrow \infty} \frac{1}{\left\|w_{i}\right\|_{A}} \eta_{w_{i}}$, there exists $i_{0} \geq 1$ such that for every $i \geq i_{0}$ and every $v \in F$ with $|v|_{A}=M$ we have

$$
\left|\frac{\left\langle v^{ \pm 1}, w_{i}\right\rangle}{\left\|w_{i}\right\|_{A}}-\langle v, \mu\rangle\right| \leq \epsilon .
$$

Hence, by the choice of $N$, for any $v \in F$ with $|v|_{A}=M$ and for any $0 \leq x \leq$ $\left\langle v^{ \pm 1}, w_{i}\right\rangle$ we have

$$
\left|\frac{x}{\left\|w_{i}\right\|_{A}}-\frac{x}{\left\|w_{i}\right\|_{A}} \frac{N}{N+C}\right| \leq \epsilon .
$$

Let $\epsilon_{1}>0$ be such that $N \epsilon_{1} \leq \epsilon / 3$.

Let $i \geq i_{0}$. Put $m=\left\|w_{i}\right\|_{A} / N$ and write $w_{i}$ as a cyclic concatenation $w_{i}=$ $y_{1} \ldots y_{m}$ of segments of length $N$. As before, for $j=1, \ldots, m$ we say that $y_{j}$ is good 
if $y_{j} \in \operatorname{supp}(\mu)$ and say that $y_{j}$ is bad otherwise. Let $m=m_{\text {bad }}+m_{\text {good }}$ where $m_{\text {bad }}$ is the number of those $j=1, \ldots, m$ such that $y_{j}$ is bad.

Since $\lim _{i \rightarrow \infty} \frac{\eta_{w_{i}}}{\left\|w_{i}\right\|_{A}}=\mu$, for any fixed freely reduced word $v \in F(A)$ of length $N$ the symmetrized frequencies $\left\langle v^{ \pm 1}, w_{i}\right\rangle_{A} /\left\|w_{i}\right\|_{A}$ of $v$ in $w_{i}$ converge to $\langle v, \mu\rangle_{A}$ as $i \rightarrow \infty$. In particular, if $v \notin \operatorname{supp}_{A}(\mu)$, these frequencies converge to zero. It follows that there exists $i_{1} \geq i_{0}$ such that for every $i \geq i_{1}$ we have $m_{\text {bad }} \leq \epsilon_{1}\left\|w_{i}\right\|_{A}$.

Let $i \geq i_{1}$. For each $j=1, \ldots, m$ such that $y_{j}$ is good, choose a path $p_{j}$ in Core $\left(\Gamma_{H}\right)$ labelled by $y_{j}$ and put $z_{j}=y_{j}$ in this case. For each $j$ such that $y_{j}$ is bad choose any reduced path $p_{j}$ of length $N$ in $\operatorname{Core}\left(\Gamma_{H}\right)$. Put $z_{j}$ to be the label of $p_{j}$ in this case.

Finally, choose reduced paths $p_{1}^{\prime}, \ldots, p_{m}^{\prime}$ in $\operatorname{Core}\left(\Gamma_{H}\right)$, of length $\leq C$ each, such that $p_{1} p_{1}^{\prime} p_{2} p_{2}^{\prime} \ldots p_{j} p_{j}^{\prime}$ defines a reduced cyclic path $\alpha$ in Core $\left(\Gamma_{H}\right)$. Let $r_{j}$ be the label of $p_{j}^{\prime}$. Then the label of the cyclic path $\alpha$ is the cyclic word $w_{i}^{\prime}$ obtained as a cyclic concatenation

$$
w_{i}^{\prime}=z_{1} r_{1} \ldots z_{m} r_{m} .
$$

Since $\left|z_{j}\right|=N$ and $\left|r_{j}\right| \leq C$, we have

and hence

$$
\left\|w_{i}\right\|_{A} \leq\left\|w_{i}^{\prime}\right\|_{A} \leq\left\|w_{i}\right\|_{A}+m C,
$$

$$
1 \leq \frac{\left\|w_{i}^{\prime}\right\|_{A}}{\left\|w_{i}\right\|_{A}} \leq 1+\frac{C}{N} .
$$

Note that by construction $w_{i}^{\prime}$ represents the $F$-conjugacy class of some $h_{i} \in H$.

Let $v \in F(A)$ be any freely reduced word of length $M$. We want to compare the frequencies of $v$ in $w_{i}$ and $w_{i}^{\prime}$. We say that an occurrence of $v^{ \pm 1}$ in $w_{i}$ is good if it lies in some good $y_{j}$ and that this occurrence is bad otherwise. Let $\left\langle v^{ \pm 1}, w_{i}\right\rangle=$ $\left\langle v^{ \pm 1}, w_{i}\right\rangle_{\text {good }}+\left\langle v^{ \pm 1}, w_{i}\right\rangle_{\text {bad }}$ where $\left\langle v^{ \pm 1}, w_{i}\right\rangle_{\text {good }}$ is the number of good occurrences of $v$ in $w_{i}$. Note that

$$
\left\langle v^{ \pm 1}, w_{i}\right\rangle_{\text {bad }} \leq N m_{\text {bad }}+M m \leq N \epsilon_{1}\left\|w_{i}\right\|_{A}+M m .
$$

Hence

$$
\frac{\left\langle v^{ \pm 1}, w_{i}\right\rangle_{\text {bad }}}{\left\|w_{i}\right\|_{A}} \leq N \epsilon_{1}+\frac{M}{N} \leq \epsilon .
$$

Thus

$$
\left|\frac{\left\langle v^{ \pm 1}, w_{i}\right\rangle_{g o o d}}{\left\|w_{i}\right\|_{A}}-\frac{\left\langle v^{ \pm 1}, w_{i}\right\rangle}{\left\|w_{i}\right\|_{A}}\right| \leq \epsilon .
$$

Similarly, we say that an occurrence of $v^{ \pm 1}$ in $w_{i}^{\prime}$ is good if it lies within some $z_{j}=y_{j}$ where $y_{j}$ is good, and that an occurrence of $v^{ \pm 1}$ in $w_{i}^{\prime}$ is bad otherwise.

We have

$$
\left\langle v^{ \pm 1}, w_{i}^{\prime}\right\rangle_{\text {bad }} \leq N m_{\text {bad }}+M m+C m \leq N \epsilon_{1}\left\|w_{i}\right\|_{A}+M m+C m .
$$

Hence

Therefore

$$
\frac{\left\langle v^{ \pm 1}, w_{i}^{\prime}\right\rangle_{\text {bad }}}{\left\|w_{i}\right\|_{A}} \leq N \epsilon_{1}+\frac{M}{N}+\frac{C}{N} .
$$

$$
\frac{\left\langle v^{ \pm 1}, w_{i}^{\prime}\right\rangle_{\text {bad }}}{\left\|w_{i}^{\prime}\right\|_{A}}=\frac{\left\langle v^{ \pm 1}, w_{i}\right\rangle_{\text {bad }}}{\left\|w_{i}\right\|_{A}} \frac{\left\|w_{i}\right\|_{A}}{\left\|w_{i}^{\prime}\right\|_{A}} \leq N \epsilon_{1}+\frac{M}{N}+\frac{C}{N} \leq \epsilon
$$

since $\left\|w_{i}\right\|_{A} \leq\left\|w_{i}^{\prime}\right\|_{A}$. 
Hence

$$
\left|\frac{\left\langle v^{ \pm 1}, w_{i}^{\prime}\right\rangle_{\text {good }}}{\left\|w_{i}^{\prime}\right\|_{A}}-\frac{\left\langle v^{ \pm 1}, w_{i}^{\prime}\right\rangle}{\left\|w_{i}^{\prime}\right\|_{A}}\right| \leq \epsilon .
$$

Note that by construction $\left\langle v, w_{i}^{\prime}\right\rangle_{\text {good }}=\left\langle v, w_{i}\right\rangle_{\text {good }}$.

Hence by $(\dagger)$

$$
\frac{\left\langle v^{ \pm 1}, w_{i}\right\rangle_{\text {good }}}{\left\|w_{i}\right\|_{A}} \geq \frac{\left\langle v^{ \pm 1}, w_{i}^{\prime}\right\rangle_{\text {good }}}{\left\|w_{i}^{\prime}\right\|_{A}}=\frac{\left\langle v^{ \pm 1}, w_{i}\right\rangle_{\text {good }}}{\left\|w_{i}\right\|_{A}} \frac{\left\|w_{i}\right\|_{A}}{\left\|w_{i}^{\prime}\right\|_{A}} \geq \frac{\left\langle v^{ \pm 1}, w_{i}\right\rangle_{\text {good }}}{\left\|w_{i}\right\|_{A}} \frac{N}{N+C} .
$$

Hence by the choice of $N$ we have

Therefore

$$
\left|\frac{\left\langle v^{ \pm 1}, w_{i}\right\rangle_{\text {good }}}{\left\|w_{i}\right\|_{A}}-\frac{\left\langle v^{ \pm 1}, w_{i}^{\prime}\right\rangle_{\text {good }}}{\left\|w_{i}^{\prime}\right\|_{A}}\right| \leq \epsilon
$$

$$
\left|\frac{\left\langle v^{ \pm 1}, w_{i}^{\prime}\right\rangle}{\left\|w_{i}^{\prime}\right\|_{A}}-\frac{\left\langle v^{ \pm 1}, w_{i}\right\rangle}{\left\|w_{i}\right\|_{A}}\right| \leq 3 \epsilon
$$

and hence

$$
\left|\frac{\left\langle v^{ \pm 1}, w_{i}^{\prime}\right\rangle}{\left\|w_{i}^{\prime}\right\|_{A}}-\langle v, \mu\rangle\right| \leq 4 \epsilon
$$

Since $w_{i}^{\prime}$ represents an element conjugate in $F$ to an element of $H$, we have verified that for any $\epsilon>0$ and any integer $M \geq 1$ condition (**) holds. This implies the statement of the proposition.

Proposition 9.11. Let $H \leq F$ be a nonabelian finitely generated subgroup. Let $T$ be an $\mathbb{R}$-tree with a very small isometric action of $F$ such that $H$ acts nontrivially on $T$. Let $T_{H}$ be the smallest $H$-invariant subtree.

Let $\mu \in \operatorname{Curr}(F)$ be such that $\operatorname{supp}(\mu) \subseteq i_{\Lambda}\left(\partial^{2} H\right)$. Then,

(1) We have

$$
\langle T, \mu\rangle \leq\left\langle T_{H},\left.\mu\right|_{H}\right\rangle \leq n_{H, F} d_{H, F}\langle T, \mu\rangle .
$$

(2) Moreover, if $H \leq F$ is malnormal, then

$$
\langle T, \mu\rangle=\left\langle T_{H},\left.\mu\right|_{H}\right\rangle .
$$

Proof. By Proposition 9.10, there exist a sequence $h_{i} \in H$ and a sequence $\lambda_{i} \geq 0$ such that

$$
\mu=\lim _{i \rightarrow \infty} \lambda_{i} \eta_{h_{i}}^{F} \text {. }
$$

We may assume that each $h_{i}$ is nontrivial and is not a proper power in $H$. For each $i$ let $1 \leq m_{i} \leq n_{H, F}$ and $h_{i, 1}, \ldots, h_{i, m_{i}} \in H$ be such that

$$
\left[h_{i}\right]_{F} \cap H=\cup_{j=1}^{m_{i}}\left[h_{i, j}\right]_{H} .
$$

Let $1 \leq d_{i} \leq d_{H, F}$ be such that in $F$ we have $h_{i}=f_{i}^{d_{i}}$ where $f_{i}$ is not a proper power in $F$.

Then by Lemma $\left.9.6 \eta_{h_{i}}^{F}\right|_{H}=d_{i} \sum_{j=1}^{m_{i}} \eta_{h_{i, j}}^{H}$. Hence

$$
\left.\mu\right|_{H}=\lim _{i \rightarrow \infty} \lambda_{i} d_{i} \sum_{j=1}^{m_{i}} \eta_{h_{i, j}}^{H} .
$$

Note that $\left\|h_{i}\right\|_{T}=\left\|h_{i, j}\right\|_{T_{H}}$. 
We have

$$
\langle T, \mu\rangle=\lim _{i \rightarrow \infty} \lambda_{i}\left\|h_{i}\right\|_{T}
$$

Also,

$$
\left\langle T_{H},\left.\mu\right|_{H}\right\rangle=\lim _{i \rightarrow \infty} \lambda_{i} d_{i} \sum_{j=1}^{m_{i}}\left\|h_{i, j}\right\|_{T_{H}}=\lim _{i \rightarrow \infty} \lambda_{i} d_{i} m_{i}\left\|h_{i}\right\|_{T} .
$$

Since $1 \leq d_{i} m_{i} \leq n_{H, F} d_{H, F},(*)$ implies that

$$
\langle T, \mu\rangle \leq\left\langle T_{H},\left.\mu\right|_{H}\right\rangle \leq n_{H, F} d_{H, F}\langle T, \mu\rangle .
$$

Moreover, if $H \leq F$ is malnormal then $n_{H, F}=d_{H, F}=1$ and hence

$$
\langle T, \mu\rangle=\left\langle T_{H},\left.\mu\right|_{H}\right\rangle,
$$

as required.

\section{The General Case}

In this section we will prove that for an arbitrary very small action of $F$ on an $\mathbb{R}$-tree $T$ the condition $\langle T, \mu\rangle=0$ implies that $\operatorname{supp}(\mu) \subseteq L^{2}(T)$.

We need the following basic fact about the structure of a general very small action (see Remark 2.1 in [CouHL2]).

Proposition-Definition 10.1 [CouHL2]. Let $F$ be a nonabelian finitely generated group with a very small minimal isometric action on an $\mathbb{R}$-tree $T$. Suppose that this action is neither discrete nor has dense orbits.

Then there exists an $F$-invariant collection $\mathcal{V}$ of disjoint closed subtrees of $T$ with the following properties:

(1) The family $\mathcal{V}$ consists of a finite number of distinct $F$-orbits of closed subtrees of $T$.

(2) For every tree $T^{\prime} \in \mathcal{V}$ the set-wise stabilizer $H=\operatorname{Stab}_{F}\left(T^{\prime}\right)$ of $T^{\prime}$ in $F$ is a finitely generated subgroup of $F$. Moreover, either $H$ acts on $T^{\prime}$ with dense orbits or $T^{\prime}$ is a single point that is fixed by $H$.

(3) Let $T_{s}$ be an $\mathbb{R}$-tree obtained from $T$ by collapsing all subtrees from $\mathcal{T}$ to points and let $q: T \rightarrow T_{s}$ be the corresponding $F$-equivariant quotient map. Then the action of $F$ on $T$ factors through to an isometric action of $F$ on $T_{s}$ that is very small, minimal and discrete.

(4) The quotient $\mathcal{Y}=T_{s} / F$ is a finite reduced graph of groups where the vertex groups correspond to setwise $F$-stabilizers of trees from $\mathcal{V}$ and where the edge-lengths correspond to distances in $T$ between $F$-orbits of the trees from $\mathcal{V}$ representing the end-points of the edge in question.

Lemma 10.2. Let $q: T \rightarrow T_{s}$ be as in Proposition-Definition 10.1. Then the following hold:

(1) For every $f \in F$ and every $p \in T$ we have $d_{T_{s}}(q(p), f q(p)) \leq d_{T}(p, f p)$.

(2) For every $f \in F$ we have $\|f\|_{T_{s}} \leq\|f\|_{T}$.

(3) For every $\mu \in \operatorname{Curr}(F)$ we have $\left\langle T_{s}, \mu\right\rangle \leq\langle T, \mu\rangle$.

(4) We have $L^{2}(T) \subseteq L^{2}\left(T_{s}\right)$. 
Proof. Part (1) follows from the definition of $q: T \rightarrow T_{s}$. Part (1) obviously implies part (2). It is also easy to see that part (2) implies parts (3) and (4). Then

Indeed, let $\mu \in \operatorname{Curr}(F)$. Write $\mu$ as $\mu=\lim _{i \rightarrow \infty} \lambda_{i} \eta_{g_{i}}$ for some $g_{i} \in F, \lambda_{i} \geq 0$.

$$
\left\langle T_{s}, \mu\right\rangle=\lim _{i \rightarrow \infty} \lambda_{i}\left\|g_{i}\right\|_{T_{s}} \leq \lim _{i \rightarrow \infty} \lambda_{i}\left\|g_{i}\right\|_{T}=\langle T, \mu\rangle,
$$

so that part (3) holds. To see that part (4) holds, fix a free basis $A$ of $F$. Suppose $v \in F(A)$ is such that $v \in\left[L^{2}(T)\right]_{A}$. Then for any $\epsilon>0$ there is a cyclically reduced $w$ with $\|w\|_{T} \leq \epsilon$ and with $v$ being a subword of $w$. Then $\|w\|_{T_{s}} \leq\|w\|_{T}$. Since $\epsilon>0$ was arbitrary, it follows that $v \in\left[L^{2}\left(T_{s}\right)\right]_{A}$, as required.

Convention 10.3. For the remainder of this section and the subsequent section, let $F, T, T_{s}$ and $Y$ be as in Proposition-Definition 10.1. For each vertex $x \in V Y$ let $G_{x} \leq F$ be a subgroup corresponding to the vertex group of $x$ of $Y$. We will denote by $V^{\prime} Y$ the set of all those $v \in V Y$ such that the vertex group of $x$ in $\mathcal{Y}$ is not cyclic.

Proposition 10.4. Let $\mu \in \operatorname{Curr}(F)$ be such that

$$
\operatorname{supp}(\mu) \subseteq L^{2}\left(T_{s}\right)=\bigcup_{x \in V^{\prime} Y} i_{\Lambda}\left(\partial^{2} G_{x}\right) .
$$

Then there exist currents $\mu_{x} \in \operatorname{Curr}(F), x \in V^{\prime} Y$, such that $\mu=\sum_{x \in V^{\prime} Y} \mu_{x}$ and such that $\operatorname{supp}\left(\mu_{x}\right) \subseteq i_{\Lambda}\left(\partial^{2} G_{x}\right)$ for every $x \in V^{\prime} Y$.

Proof. There is a finite collection of nontrivial elements $g_{1}, \ldots, g_{p} \in F$ such that if for some $x_{1}, x_{2} \in V^{\prime} Y, x_{1} \neq x_{2}$, and some $f_{1}, f_{2} \in G$ we have $f_{1} G_{x_{1}} f_{1}^{-1} \cap f_{2} G_{x_{2}} f_{2}^{-1} \neq 1$, then $f_{1} G_{x_{1}} f_{1}^{-1} \cap f_{2} G_{x_{2}} f_{2}^{-1}$ is a cyclic group generated by a conjugate of the power of some $g_{i}$ in $F$. Indeed, suppose that $H=f_{1} G_{x_{1}} f_{1}^{-1} \cap f_{2} G_{x_{2}} f_{2}^{-1} \neq 1$. Then $H$ fixes a segment in $T_{s}$ joining a vertex $v_{1}$ of $T_{s}$ projecting to $x_{1}$ with a vertex $v_{2}$ of $T_{s}$ projecting to $x_{2}$. Hence $H$ fixes an edge of $T_{s}$ adjacent to $v_{1}$. This implies that $H$ is conjugate to a subgroup of the edge-group for an edge adjacent to $x_{1}$ in $\mathcal{Y}$. Thus we can take $g_{1}, \ldots, g_{p} \in F$ to be the generators of the nontrivial edge-groups of $\mathcal{Y}$.

One can then show that if $x_{1}, x_{2} \in V^{\prime} Y, x_{1} \neq x_{2}$ and a point $(\xi, \zeta) \in \partial^{2} F$ belongs to $i_{\Lambda}\left(\partial^{2} G_{x_{1}}\right) \cap i_{\Lambda}\left(\partial^{2} G_{x_{2}}\right)$ then for some $1 \leq i \leq p$ the point $(\xi, \zeta)$ is an $F$-translate of $\left(g_{i}^{-\infty}, g_{i}^{\infty}\right)$ or of $\left(g_{i}^{\infty}, g_{i}^{-\infty}\right)$. To see this, choose a free basis $A$ of $F$ and suppose that $(\xi, \zeta) \in i_{\Lambda}\left(\partial^{2} G_{x_{1}}\right) \cap i_{\Lambda}\left(\partial^{2} G_{x_{2}}\right)$. Then the bi-infinite geodesic joining $\xi$ to $\zeta$ in the Cayley graph $X(F, A)$ is labelled by a bi-infinite freely reduced word $w$ that can be read along some bi-infinite paths in cores of the Stallings subgroup graphs $\Gamma_{G_{x_{1}}}$ and $\Gamma_{G_{x_{2}}}$. Therefore (see $\left.[\mathrm{KaM}]\right) w$ can be read along a bi-infinite path in a connected component of the "Stallings product graph" $\Gamma_{G_{x_{1}}} \times \Gamma_{G_{x_{2}}}$ (also known as the push-out of $\Gamma_{G_{x_{1}}}$ and $\Gamma_{G_{x_{2}}}$ ). Hence (again see $[\mathrm{KaM}]$ ) the bi-infinite word $w$ is readable along some path in the core of the Stallings subgroup graph for a subgroup of the form $f_{1} G_{x_{1}} f_{1}^{-1} \cap f_{2} G_{x_{2}} f_{2}^{-1}$ for some $f_{1}, f_{2} \in F$. Therefore $w$ is an infinite power of some $g_{i}$, as required.

For $1 \leq i \leq p$ put $\lambda_{i}=\mu\left(\left\{\left(g_{i}^{-\infty}, g_{i}^{\infty}\right)\right\}\right)=\mu\left(\left\{\left(g_{i}^{\infty}, g_{i}^{-\infty}\right)\right\}\right)$. Thus $\lambda_{i} \geq 0$. 
Hence we can represent $\mu$ as

$$
\mu=\nu+\sum_{i=1}^{p} \lambda_{i} \eta_{g_{i}}
$$

where $\nu \in \operatorname{Curr}(F)$ is a geodesic current, and where for every $1 \leq i \leq p$ the current $\nu$ has no atom at $\left(g_{i}^{-\infty}, g_{i}^{\infty}\right)$, and hence it has no atom at every $F$-translate of $\left(g_{i}^{-\infty}, g_{i}^{\infty}\right)$ or of $\left(g_{i}^{\infty}, g_{i}^{-\infty}\right)$. Here by saying that a measure has no atom at a particular point we mean that the measure of a singleton consisting of that point is equal to zero.

For the current $\nu$ the statement of the proposition is obvious. Indeed, for $S \subseteq \partial^{2} F$ and for $x \in V^{\prime} Y$ put

$$
\nu_{x}(S)=\nu\left(S \cap i_{\Lambda}\left(\partial^{2} G_{x}\right)\right) .
$$

It is not hard to check that for every $x \in V^{\prime} Y$ we have $\nu_{x} \in \operatorname{Curr}(F)$ and $\operatorname{supp}\left[\nu_{x}\right] \subseteq i_{\Lambda}\left(\partial^{2} G_{x}\right)$, and that $\nu=\sum_{x \in V^{\prime} Y} \nu_{x}$.

Note that for every $1 \leq i \leq p$ there is some (not necessarily unique) $x(i)=$ $x \in V^{\prime} Y$ such that $\operatorname{supp}\left(\eta_{g_{i}}\right) \subseteq i_{\Lambda}\left(\partial^{2} G_{x}\right)$.

For every $x \in V^{\prime} Y$ put $\mu_{x}$ to be the sum of $\nu_{x}$ and all those $\lambda_{i} \eta_{g_{i}}$ for which $x(i)=x$. Then $\mu=\sum_{x \in V^{\prime} Y} \mu_{x}$ and $\operatorname{supp}\left(\mu_{x}\right) \subseteq i_{\Lambda}\left(\partial^{2} G_{x}\right)$ for every $x \in V^{\prime} Y$, as required.

Note that the decomposition $\mu=\sum_{x \in V^{\prime} Y} \mu_{x}$ in Proposition 10.4 is, in general, non-canonical.

Proposition 10.5. Let $H \leq F=F(A)$ be a finitely generated subgroup and let $\mu \in \operatorname{Curr}_{H}(F)$.

Then $\operatorname{supp}(\mu) \subseteq i_{\Lambda}\left(\operatorname{supp}\left(\left.\mu\right|_{H}\right)\right)$.

Proof. Let $\Gamma_{H}$ be the Stallings subgroup graph of $H$ with respect to $A$ and let $\Delta_{H}=\operatorname{Core}\left(\Gamma_{H}\right)$. Note that the minimal $H$-invariant subtree $X_{H} \subseteq X(F, A)$ is a copy of $\widetilde{\Delta_{H}}$ in $X(F, A)$.

By conjugating $H$ if necessary, without loss of generality we may assume that in fact $\Delta_{H}=\Gamma_{H}$, so that the base-vertex $y \in V \Gamma_{H}$ is a vertex of $\Delta_{H}$. Then $\left(\Gamma_{H}, y\right)$ provides a canonical isomorphism (simplicial chart) $\alpha: H \rightarrow \pi_{1}\left(\Gamma_{H}, y\right)$. This isomorphism in turn provides a canonical $H$-equivariant identification of $\partial H$ with $\partial \widetilde{\Gamma_{H}}=\partial X_{H}$ and a corresponding $H$-equivariant identification of $\partial^{2} H$ with $\partial^{2} X_{H}$. From this point on we will assume that these identifications are made without additional comment.

Recall that the lamination $L:=i_{\Lambda}\left(\operatorname{supp}\left(\left.\mu\right|_{H}\right)\right) \in \Lambda^{2}(F)$ has the form

$$
L=\overline{\cup_{f \in F} f \operatorname{supp}\left(\left.\mu\right|_{H}\right)} \text {. }
$$

Let $v \in F(A)$ be such that $v \in \operatorname{supp}_{A}(\mu)$, so that $\langle v, \mu\rangle_{A}>0$. We need to show that $v \in L_{A}$.

Fix a segment $[x, y]$ in $X_{H}$ labelled by $v$. 
Since $\operatorname{supp}(\mu) \subseteq i_{\Lambda}\left(\partial^{2} H\right)$, we know that

$$
\begin{aligned}
\langle v, \mu\rangle=\mu\left(\mathrm{Cyl}_{X}([x, y])\right) & =\mu\left(\mathrm{Cyl}_{X}([x, y]) \cap\left(\cup_{f \in F} f \partial^{2} H\right)\right) \\
& =\bigcup_{f \in F}\left(\operatorname{Cyl}_{X}([x, y]) \cap f \partial^{2} H\right)>0 .
\end{aligned}
$$

Since $\mu$ is countably additive, there is some $f \in F$ such that

$$
\mu\left(\mathrm{Cyl}_{X}([x, y]) \cap f \partial^{2} H\right)=\mu\left(f^{-1} \mathrm{Cyl}_{X}([x, y]) \cap \partial^{2} H\right)>0 .
$$

It is easy to see that if $[x, y]$ is not contained in $f X_{H}$ then $\operatorname{Cyl}_{X}([x, y])$ and $f \partial^{2} H$ are disjoint. Hence we have that $[x, y] \subseteq f X_{H}$ and that $[x, y]$ is labelled by $v$ in $X=X(F, H)$. Thus there is a reduced path $p$ in $\Delta_{H}$ labelled by $v$ such that $\left[x^{\prime}, y^{\prime}\right]:=f^{-1}[x, y] \subseteq X_{H}=\widetilde{\Delta}_{H}$ is a lift of $p$ in $\widetilde{\Delta}_{H}$. Note that $f^{-1} \operatorname{Cyl}_{X}([x, y])=$ $\mathrm{Cyl}_{X}\left(\left[x^{\prime}, y^{\prime}\right]\right)$ so that $\mu\left(\mathrm{Cyl}_{X}\left(\left[x^{\prime}, y^{\prime}\right]\right) \cap \partial^{2} H\right)>0$. It is easy to see that

$$
\mathrm{Cyl}_{X}\left(\left[x^{\prime}, y^{\prime}\right]\right) \cap \partial^{2} H=\mathrm{Cyl}_{X_{H}}\left(\left[x^{\prime}, y^{\prime}\right]\right) \subseteq \partial^{2} H .
$$

Therefore by definition of $\left.\mu\right|_{H}$ we have

$$
\left.\mu\right|_{H}\left(\mathrm{Cyl}_{X_{H}}\left(\left[x^{\prime}, y^{\prime}\right]\right)\right)=\mu\left(\mathrm{Cyl}_{X_{H}}\left(\left[x^{\prime}, y^{\prime}\right]\right)\right)=\mu\left(\mathrm{Cyl}_{X}\left(\left[x^{\prime}, y^{\prime}\right]\right) \cap \partial^{2} H\right)>0 .
$$

Thus $\left\langle p,\left.\mu\right|_{H}\right\rangle_{\alpha}>0$ and hence $p \in \operatorname{supp}_{\alpha}\left(\left.\mu\right|_{H}\right)$. Since $v \in F(A)$ is the label of $p$, it follows that $v \in L_{A}$ where $L=i_{\Lambda}\left(\left.\operatorname{supp} \mu\right|_{H}\right)$, as required.

Proposition 10.6. Let $x \in V^{\prime} Y$ and let $\mu \in \operatorname{Curr}(F)$ be such that $\operatorname{supp}(\mu) \subseteq i_{\Lambda}\left(\partial^{2} G_{x}\right)$ and $\langle T, \mu\rangle=0$. Then $\operatorname{supp}(\mu) \subseteq L^{2}(T)$.

Proof. Denote $H=G_{x}$. Fix a free basis $A$ of $F$ and a free basis $B$ of $H$. If $H$ fixes a vertex of $T$ then $i_{\Lambda}\left(\partial^{2} H\right) \subseteq L^{2}(T)$ and the statement is obvious. Suppose that $H$ acts nontrivially on $T$ and let $T_{H}$ be the minimal $H$-invariant subtree of $T$. Then $H$ acts on $T_{H}$ with dense orbits.

Since $\operatorname{supp}(\mu) \subseteq i_{\Lambda}\left(\partial^{2} H\right)$ and $\langle T, \mu\rangle=0$, Proposition 9.11 implies that $\left\langle T_{H},\left.\mu\right|_{H}\right\rangle$ $=0$. Since $H$ acts on $T_{H}$ with dense orbits, we know by Proposition 6.1 that $\operatorname{supp}\left(\left.\mu\right|_{H}\right) \subseteq L^{2}\left(T_{H}\right) \subseteq \partial^{2} H$.

Since $\operatorname{supp}(\mu) \subseteq i_{\Lambda}\left(\partial^{2} H\right)$, Proposition 10.5 implies that $\operatorname{supp}(\mu) \subseteq i_{\Lambda}\left(\operatorname{supp}\left(\left.\mu\right|_{H}\right)\right)$ and hence

$$
\operatorname{supp}(\mu) \subseteq i_{\Lambda}\left(L^{2}\left(T_{H}\right)\right) .
$$

It is easy to see that $i_{\Lambda}\left(L^{2}\left(T_{H}\right)\right) \subseteq L^{2}(T)$ and the statement of the proposition follows.

Theorem 10.7. Let $F$ be a finitely generated nonabelian free group with a very small isometric minimal action on an $\mathbb{R}$-tree $T$. Let $\mu \in \operatorname{Curr}(F)$ be such that $\langle T, \mu\rangle=0$. Then $\operatorname{supp}(\mu) \subseteq L^{2}(T)$.

Proof. If $F$ acts on $T$ with dense orbits or if the action is simplicial, the statement of the theorem follows from Proposition 6.1 and Proposition 8.4. Suppose neither of these two situations occurs. Let $q: T \rightarrow T_{s}=\widetilde{\mathcal{Y}}$ be as in Proposition-Definition 10.1. 
By Proposition 10.4 we can decompose $\mu$ as

$$
\mu=\sum_{x \in V^{\prime} Y} \mu_{x},
$$

where each $\mu_{x} \in \operatorname{Curr}(F)$ satisfies $\operatorname{supp}\left(\mu_{x}\right) \subseteq i_{\Lambda}\left(\partial^{2} H\right)$.

We have

$$
0=\langle T, \mu\rangle=\sum_{x \in V^{\prime} Y}\left\langle T, \mu_{x}\right\rangle
$$

and hence $\left\langle T, \mu_{x}\right\rangle=0$ for each $x \in V^{\prime} Y$.

Therefore by Proposition 10.6 we have $\operatorname{supp}\left(\mu_{x}\right) \subseteq L^{2} T$ for every $x \in V X$.

Since, obviously, $\operatorname{supp}(\mu)=\cup_{x \in V^{\prime} Y} \operatorname{supp}\left(\mu_{x}\right)$, it follows that $\operatorname{supp}(\mu) \subseteq L^{2}(T)$, as required.

Lemma 10.8. Let $F=F(A)$ be a finitely generated nonabelian free group. Let $T$ be an $\mathbb{R}$-tree with a very small nontrivial minimal isometric action of $F$ on $T$. Then $L^{2}(T) \neq \partial^{2} F$.

Proof. Put $L=L^{2}(T)$. We need to show that there exists $v \in F(A)$ such that $v \notin L_{A}$.

Let $p \in T$. By Proposition 4.2, there is $C<\infty$ such that $B B T_{T, p}(A)<C$.

Since the action of $F$ on $T$ is nontrivial, there exists a freely reduced $v \in F(A)$ such that $\|v\|_{T} \geq 4 C+2$. Hence $d_{T}(p, v p) \geq\|v\|_{T} \geq 4 C+2$. Suppose that $v \in L_{A}$. Then there exists a cyclically reduced word $w$ in $F(A)$ such that $v$ is an initial segment of $w$ and such that $\|w\|_{T} \leq 1$. We can write $w$ as a reduced product $w=v u$. By Lemma 4.3 we have

$$
d_{T}(p, w p) \geq d_{T}(p, v p)+d_{T}(p, u p)-2 C \geq\|v\|_{T}-2 C .
$$

Since $w$ is cyclically reduced, Lemma 4.3 also implies that

$$
\|w\|_{T} \geq d_{T}(p, w p)-2 C \geq\|v\|_{T}-4 C \geq 2 .
$$

This contradicts our assumption that $\|w\|_{T} \leq 1$. Thus $v \notin L_{A}$.

Corollary 10.9. Let $\mu \in \operatorname{Curr}(F)$ be a current with full support. Then for every very small action of $F$ on an $\mathbb{R}$-tree $T$ we have $\langle T, \mu\rangle>0$.

Proof. Suppose there is some very small $T$ such that $\langle\mu, T\rangle=0$. By Theorem 10.7 it follows that $\operatorname{supp}(\mu) \subseteq L^{2}(T)$. By Lemma 10.8 this contradicts our assumption that $\mu$ has full support.

\section{Length Compactness for Currents with Full Support}

DeFinition 11.1 (Automorphic length spectrum of a current). Let $T \in \overline{c v}(F)$ and $\mu \in \operatorname{Curr}(F)$. The automorphic length spectrum of $\mu$ with respect to $T$ is the set

$$
\mathcal{S}_{T}(\mu):=\{\langle T, \phi \mu\rangle: \phi \in \operatorname{Out}(F)\} \subseteq \mathbb{R} .
$$

Note that by $\operatorname{Out}(F)$-invariance of the intersection form, we always have $\langle T, \phi \mu\rangle=$ $\left\langle\phi^{-1} T, \mu\right\rangle$. Hence

$$
\mathcal{S}_{T}(\mu):=\{\langle\phi T, \mu\rangle: \phi \in \operatorname{Out}(F)\} .
$$


Theorem 11.2. Let $\mu \in \operatorname{Curr}(F)$ be a current with full support and let $T \in \operatorname{cv}(F)$. Then,

(1) For any $C>0$ the set

$$
\{\phi \in \operatorname{Out}(F):\langle T, \phi \mu\rangle \leq C\}
$$

is finite.

(2) The set $\mathcal{S}_{T}(\mu)$ is a discrete subset of $\mathbb{R}_{\geq 0}$.

(3) Suppose $\phi_{n} \in \operatorname{Out}(F)$ is an infinite sequence of distinct elements such that for some $\lambda_{n} \geq 0$ and some $\mu^{\prime} \in \operatorname{Curr}(F)$ we have $\lim _{n \rightarrow \infty} \lambda_{n} \phi_{n} \mu=\mu^{\prime}$. Then $\lim _{n \rightarrow \infty} \lambda_{n}=0$.

Proof. It is obvious that (1) implies (2).

To see that (1) holds, suppose that for some $C>0$ there exists an infinite sequence of distinct elements $\phi_{n} \in \operatorname{Out}(F)$ such that for every $n \geq 1$ we have $\left\langle T, \phi_{n} \mu\right\rangle \leq C$. By rescaling $T$, we may assume that the graph $T / F$ has volume 1 , that is $T \in C V(F)$.

Since $\overline{C V}(F)=C V(F) \cup \partial C V(F)$ is compact, after passing to a subsequence we may assume that $\lim _{n \rightarrow \infty}\left[\phi_{n}^{-1} T\right]=\left[T_{\infty}\right]$ in $\overline{C V}(F)$ for some $T_{\infty} \in \overline{c v}(F)$. Thus there is a sequence $c_{n} \geq 0$ such that

$$
\lim _{n \rightarrow \infty} c_{n} \phi_{n}^{-1} T=T_{\infty}
$$

in $\overline{c v}(F)$. Moreover, since all $\phi_{n} \in \operatorname{Out}(F), n \geq 1$ are distinct and the action of $\operatorname{Out}(F)$ on $C V(F)=\mathbb{P} c v(F)$ is properly discontinuous, we have $\left[T_{\infty}\right] \in \partial C V(F)$. This implies that $\lim _{n \rightarrow \infty} c_{n}=0$.

Indeed, suppose not. Then, after passing to a subsequence, we may assume that $c_{n} \geq c>0$ for every $n \geq 1$. Since $T_{\infty} \notin c v(F)$, there are nontrivial elements in $F$ acting on $T_{\infty}$ with arbitrary small translation length. That is, there exists a sequence $g_{i} \in F, g_{i} \neq 1$ such that $\lim _{i \rightarrow \infty}\left\|g_{i}\right\|_{T_{\infty}}=0$. Recall that by definition of the left action of $\operatorname{Out}(F)$ on $\overline{c v}(F)$, we have $\|g\|_{\phi_{n}^{-1} T}=\left\|\phi_{n}(g)\right\|_{T}$ for every $g \in F$ and every $n \geq 1$. Then (\$) implies that there is a sequence $n_{i} \geq 1$ with $\lim _{i \rightarrow \infty} n_{i}=\infty$ such that

$$
0=\lim _{i \rightarrow \infty} c_{n_{i}}\left\|g_{i}\right\|_{\phi_{n_{i}}^{-1} T}=\lim _{i \rightarrow \infty} c_{n_{i}}\left\|\phi_{n_{i}} g_{i}\right\|_{T} .
$$

On the other hand, since the action of $F$ on $T$ is free and simplicial, there is some $\delta>0$ such that for every $f \in F, f \neq 1$ we have $\|f\|_{T} \geq \delta$. Hence for every $i \geq 1$

$$
c_{n_{i}}\left\|\phi_{n_{i}} g_{i}\right\|_{T} \geq c \delta>0,
$$

yielding a contradiction. Thus indeed $\lim _{n \rightarrow \infty} c_{n}=0$.

By $\operatorname{Out}(F)$-invariance of the intersection form we have

$$
0 \leq\left\langle c_{n} \phi_{n}^{-1} T, \mu\right\rangle=c_{n}\left\langle\phi_{n}^{-1} T, \mu\right\rangle=c_{n}\left\langle T, \phi_{n} \mu\right\rangle \leq c_{n} C \rightarrow_{n \rightarrow \infty} 0 .
$$

Therefore, by the continuity of the intersection form [KaLu2], we have

$$
0=\lim _{n \rightarrow \infty}\left\langle c_{n} \phi_{n}^{-1} T, \mu\right\rangle=\left\langle T_{\infty}, \mu\right\rangle .
$$

However, this contradicts the conclusion of Corollary 10.9 since by assumption $\mu$ has full support. Thus part (1) is established. 
We now show that (1) implies (3). Let $\lambda_{n}, \phi_{n}, \mu^{\prime}$ be as in (3). Part (1) implies that $\lim _{n \rightarrow \infty}\left\langle T, \phi_{n} \mu\right\rangle=\infty$. On the other hand,

$$
\lim _{n \rightarrow \infty} \lambda_{n}\left\langle T, \phi_{n} \mu\right\rangle=\lim _{n \rightarrow \infty}\left\langle T, \lambda_{n} \phi_{n} \mu\right\rangle=\left\langle T, \mu^{\prime}\right\rangle<\infty .
$$

This implies that $\lim _{n \rightarrow \infty} \lambda_{n}=0$, as required.

\section{Unique Ergodicity}

Recall that an element $\phi \in \operatorname{Out}(F)$ is called reducible if there exists a free product decomposition $F=C_{1} * \ldots C_{k} * F^{\prime}$, where $k \geq 1$ and $C_{i} \neq\{1\}$, such that $\phi$ permutes the conjugacy classes of subgroups $C_{1}, \ldots, C_{k}$ in $F$. An element $\phi \in \operatorname{Out}(F)$ is called irreducible if it is not reducible.

Definition 12.1. An element $\phi \in \operatorname{Out}(F)$ is said to be irreducible with irreducible powers or an iwip for short, if for every $n \geq 1 \phi^{n}$ is irreducible (sometimes such automorphisms are also called fully irreducible). Thus $\phi \in \operatorname{Aut}(F)$ is an iwip if and only if no positive power of $\phi$ preserves the conjugacy class of a proper free factor of $F$. An element $\phi \in \operatorname{Aut}(F)$ is atoroidal that is, if there does not exist a nontrivial conjugacy class in $F$ that is fixed by some positive power of $\phi$.

Let $\phi \in \operatorname{Out}(F)$ be an atoroidal iwip. It is known, by the work of Reiner Martin in the case of currents and by the result of Levitt and Lustig in the case of $\overline{C V}(F)$ that the (left) action of $\phi$ has "North-South" dynamics on both $\mathbb{P} \operatorname{Curr}(F)$ and $\overline{C V}(F)$.

Proposition 12.2. Let $\phi \in \operatorname{Out}(F)$ be an atoroidal iwip. Then the following hold:

(1) [LevL] The action of $\phi$ on $\overline{C V}(F)$ has exactly two distinct fixed points $\left[T_{+}\right],\left[T_{-}\right]$and, moreover, for any $[T] \in \overline{C V}(F),[T] \neq\left[T_{-}\right]$we have $\lim _{n \rightarrow \infty} \phi^{n}[T]=\left[T_{+}\right]$and for any $[T] \in \overline{C V}(F),[T] \neq\left[T_{+}\right]$we have $\lim _{n \rightarrow \infty} \phi^{-n}[T]=\left[T_{-}\right]$.

(2) $[\mathrm{M}]$ The action of $\phi$ on $\mathbb{P} \operatorname{Curr}(F)$ has exactly two distinct fixed points $\left[\mu_{+}\right],\left[\mu_{-}\right]$and, moreover, for any $[\mu] \in \mathbb{P} \operatorname{Curr}(F),[\mu] \neq\left[\mu_{-}\right]$we have $\lim _{n \rightarrow \infty} \phi^{n}[\mu]=\left[\mu_{+}\right]$and for any $[\mu] \in \mathbb{P} \operatorname{Curr}(F),[\mu] \neq\left[\mu_{+}\right]$we have $\lim _{n \rightarrow \infty} \phi^{-n}[\mu]=\left[\mu_{-}\right]$.

(3) $[\mathrm{KaLu2}]$ We have $\left\langle T_{+}, \mu_{+}\right\rangle=\left\langle T_{-}, \mu_{-}\right\rangle=0$ and $\left\langle T_{+}, \mu_{-}\right\rangle>0,\left\langle T_{-}, \mu_{-}\right\rangle>0$.

Here we prove that $\mu_{+}$is uniquely ergodic in the following sense:

Theorem 12.3. Let $\phi \in \operatorname{Out}(F), \mu_{ \pm}, T_{ \pm}$be as in Proposition 12.2. If $[\mu] \in \mathbb{P} \operatorname{Curr}(F)$ is such that $\operatorname{supp}(\mu) \subseteq \operatorname{supp}\left(\mu_{+}\right)$then $[\mu]=\left[\mu_{+}\right]$.

Proof. Note that since $\phi$ fixes $\left[T_{+}\right]$, we have $\phi T_{+}=\lambda T_{+}$for some $\lambda>0$. Suppose that $\operatorname{supp}(\mu) \subseteq \operatorname{supp}\left(\mu_{+}\right)$but $[\mu] \neq\left[\mu_{+}\right]$. Since $\left\langle T_{+}, \mu_{+}\right\rangle=0$, Theorem 1.1 implies that $\operatorname{supp}\left(\mu_{+}\right) \subseteq L^{2}\left(T_{+}\right)$. Since $\operatorname{supp}(\mu) \subseteq \operatorname{supp}\left(\mu_{+}\right) \subseteq L^{2}\left(T_{+}\right)$, Theorem 1.1 also implies that $\left\langle T_{+}, \mu\right\rangle=0$. Since $[\mu] \neq\left[\mu_{+}\right]$we have $\lim _{n \rightarrow \infty} \phi^{-n}[\mu]=\left[\mu_{-}\right]$, so that for some sequence $c_{n}>0$ we have $\lim _{n \rightarrow \infty} c_{n} \phi^{-n} \mu=\mu_{-}$in $\operatorname{Curr}(F)$. Hence for any $n \geq 1$

$$
\left\langle T_{+}, c_{n} \phi^{-n} \mu\right\rangle=\left\langle\phi^{n} T_{+}, c_{n} \mu\right\rangle=\left\langle\lambda^{n} T_{+}, c_{n} \mu\right\rangle=\lambda^{n} c_{n}\left\langle T_{+}, \mu\right\rangle=0 .
$$


Therefore, by continuity of the intersection form,

$$
0=\lim _{n \rightarrow \infty}\left\langle T_{+}, c_{n} \phi^{-n} \mu\right\rangle=\left\langle T_{+}, \lim _{n \rightarrow \infty} c_{n} \phi^{-n} \mu\right\rangle=\left\langle T_{+}, \mu_{-}\right\rangle,
$$

which contradicts part (3) of Proposition 12.2.

By a similar argument we obtain a dual statement for $T_{+}$.

Theorem 12.4. Let $\phi \in \operatorname{Out}(F), \mu_{ \pm}, T_{ \pm}$be as in Proposition 12.2. Let $[T] \in \overline{C V}(F)$ be such that $L^{2}\left(T_{+}\right) \subseteq L^{2}(T)$. Then $[T]=\left[T_{+}\right]$.

Proof. Note that since $\phi$ fixes $\left[\mu_{+}\right]$, there is $r>0$ such that $\phi \mu_{+}=r \mu_{+}$. Suppose that $[T] \in \overline{C V}(F)$ be such that $L^{2}\left(T_{+}\right) \subseteq L^{2}(T)$ but that $[T] \neq\left[T_{+}\right]$. We have $\operatorname{supp}\left(\mu_{+}\right) \subseteq L^{2}\left(T_{+}\right) \subseteq L^{2}(T)$ and therefore by Theorem 1.1 we have $\left\langle T, \mu_{+}\right\rangle=0$. Since $[T] \neq\left[T_{+}\right]$, we have $\lim _{n \rightarrow \infty} \phi^{-n}[T]=\left[T_{-}\right]$, so that for some sequence $c_{n}>0$ we have $\lim _{n \rightarrow \infty} c_{n} \phi^{-n} T=T_{-}$in $\overline{c v}(F)$. Hence for any $n \geq 1$

$$
\left\langle c_{n} \phi^{-n} T, \mu_{+}\right\rangle=\left\langle c_{n} T, \phi^{n} \mu_{+}\right\rangle=\left\langle c_{n} T, r^{n} \mu_{+}\right\rangle=r^{n} c_{n}\left\langle T, \mu_{+}\right\rangle=0 .
$$

Therefore, by continuity of the intersection form

$$
0=\lim _{n \rightarrow \infty}\left\langle c_{n} \phi^{-n} T, \mu_{+}\right\rangle=\left\langle\lim _{n \rightarrow \infty} c_{n} \phi^{-n} T, \mu_{+}\right\rangle=\left\langle T_{-}, \mu_{+}\right\rangle,
$$

which contradicts part (3) of Proposition 12.2.

Corollary 12.5. Let $\phi \in \operatorname{Out}(F), \mu_{ \pm}, T_{ \pm}$be as in Proposition 12.2.

(1) Let $T \in \overline{c v}(F)$. Then $\left\langle T, \mu_{+}\right\rangle=0$ if and only if $[T]=\left[T_{+}\right]$.

(2) Let $\mu \in \operatorname{Curr}(F), \mu \neq 0$. Then $\left\langle T_{+}, \mu\right\rangle=0$ if and only if $[\mu]=\left[\mu_{+}\right]$.

Proof. (1) We already know that $\left\langle T_{+}, \mu_{+}\right\rangle=0$ and that $\operatorname{supp}\left(\mu_{+}\right) \subseteq L^{2}\left(T_{+}\right)$. Suppose $\left\langle T, \mu_{+}\right\rangle=0$ for some $T \in \overline{c v}(F)$. Suppose that $[T] \neq\left[T_{+}\right]$. Then $\lim _{n \rightarrow \infty} \phi^{-n}[T]=\left[T_{-}\right]$, so that $\lim _{n \rightarrow \infty} c_{n} \phi^{-n} T=T_{-}$for some $c_{n}>0$. We have

$$
\left\langle c_{n} \phi^{-n} T, \mu_{+}\right\rangle=\left\langle c_{n} T, \phi^{n} \mu_{+}\right\rangle=\left\langle c_{n} T, r^{n} \mu_{+}\right\rangle=c_{n} r^{n}\left\langle T, \mu_{+}\right\rangle=0 .
$$

Since $\lim _{n \rightarrow \infty} c_{n} \phi^{-n} T=T_{-}$, the continuity of the intersection form implies that $\left\langle T_{-}, \mu_{+}\right\rangle=0$, yielding a contradiction with part (3) of Proposition 12.2.

The proof of part (2) is essentially symmetric and we omit the details.

As noted in the above argument, we do know that $\operatorname{supp}\left(\mu_{+}\right) \subseteq L^{2}\left(T_{+}\right)$, but even in this particular case it can happen that the inclusion is a strict one.

One can check, by directly comparing the definitions, that, under the assumptions of Proposition 12.2, $\operatorname{supp}\left(\mu_{+}\right)$is equal to the "stable lamination" of $\phi$ in the sense of [BeFH1]. The reason for the potential inequality $\operatorname{supp}\left(\mu_{+}\right) \neq L^{2}\left(T_{+}\right)$comes from the possibility that $(\xi, \zeta),(\zeta, \omega) \in \operatorname{supp}\left(\mu_{+}\right)$but $(\xi, \omega) \notin \operatorname{supp}\left(\mu_{+}\right)$, while this type of behavior is by definition impossible in $L^{2}\left(T_{+}\right)$.

We believe that that for any atoroidal iwip automorphism $L^{2}\left(T_{+}\right)$is obtained from the stable lamination of $\phi$ via the operation of "diagonal closure" as indicated above.

\section{Filling Elements, Filling Currents and Bounded Translation Equivalence}

Definition 13.1. Let $\mu \in \operatorname{Curr}(F)$. We say that $\mu$ fills $F$ if for every very small action of $F$ on an $\mathbb{R}$-tree $T$ we have $\langle T, \mu\rangle>0$. 
Similarly, we say that an element $g \in F$ fills $F$ if for every very small action of $F$ on an $\mathbb{R}$-tree $T$ we have $\|g\|_{T}>0$. Thus $g$ fills $F$ if and only if $\eta_{g}$ fills $F$.

Corollary 10.9 says that every current $\mu \in \operatorname{Curr}(F)$ with full support fills $F$.

Proposition 13.2. Let $\mu \in \operatorname{Curr}(F)$ be a current with full support. Let $\mu_{n} \in \operatorname{Curr}(F)$ be a sequence such that $\lim _{n \rightarrow \infty} \mu_{n}=\mu$. Then there is $n_{0} \geq 1$ such that for every $n \geq n_{0}$ the current $\mu_{n}$ fills $F$.

Proof. Suppose the statement of the proposition fails. Then there exists a sequence $n_{i}$ with $\lim _{i \rightarrow \infty} n_{i}=\infty$ and a sequence of very small $\mathbb{R}$-trees $T_{i}$ such that $\left\langle T_{i}, \mu_{n_{i}}\right\rangle=0$. Since $C V(F) \cup \partial C V(F)$ is compact, there exists a sequence $r_{i} \geq 0$ and a very small action of $F$ on an $\mathbb{R}$-tree $T$ such that $\lim _{i \rightarrow \infty} r_{i} T_{i}=T$. Note that we have

$$
\left\langle r_{i} T_{i}, \mu_{n_{i}}\right\rangle=r_{i}\left\langle T_{i}, \mu_{n_{i}}\right\rangle=0 .
$$

By the continuity of the intersection form on the closure of the non-projectivized outer space (see [KaLu2]), this implies that

$$
\langle T, \mu\rangle=0 \text {. }
$$

This contradicts the fact that, by Corollary 10.9, $\mu$ fills $F$.

Proposition 13.2 immediately implies

Corollary 13.3. Let $\mu \in \operatorname{Curr}(F)$ be a current with full support. Let $\lambda_{n} \geq 0$ and $g_{n} \in F$ be such that $\lim _{n \rightarrow \infty} \lambda_{n} \eta_{g_{n}}=\mu$. Then there is $n_{0} \geq 1$ such that for every $n \geq n_{0}$ the element $g_{n}$ fills $F$.

Notation 13.4. Let $F$ be a finitely generated free group and let $A$ be a free basis of $F$. Let $\xi \in \partial F$. We represent $\xi$ by a right-infinite freely reduced word $x_{1} x_{2} \ldots x_{n} \ldots$, where $x_{i} \in A^{ \pm 1}$, labelling the geodesic ray from 1 to $\xi$ in the Cayley graph $X(F, A)$. For every $n \geq 1$ we denote by $\xi_{A}(n)$ the element of $F$ represented by the initial segment of this ray of length $n$, that is $\xi_{A}(n)=x_{1} \ldots x_{n} \in F$.

Definition 13.5 (Uniform measure corresponding to a free basis). Let $A$ be a free basis of $F$ and let $k \geq 2$ be the rank of $F$. For a nontrivial freely reduced word $v \in F(A)$ let $\mathrm{Cyl}_{A}(v)$ be the set of all $\xi \in \partial F$ such that $v$ is an initial segment of $\xi$, when $\xi$ is realized as a geodesic ray with origin $1 \in F$ in the Cayley graph $X(F, A)$.

The uniform measure on $\partial F$, corresponding to $A$, denoted $\mu_{A}$, is a Borel probability measure on $\partial F$, such that for every nontrivial freely reduced word $v \in F(A)$ we have

$$
\mu_{A}\left(\mathrm{Cyl}_{A}(v)\right)=\frac{1}{2 k(2 k-1)^{n-1}},
$$

where $n=|v|_{A}$.

Informally, a $\mu_{A}$-random point $\xi \in \partial F$ corresponds to a "random" right-infinite freely reduced word over $A^{ \pm 1}$. We refer the reader to [Ka4], [KKS], [KaN] for a more detailed discussion regarding the uniform measure $\mu_{A}$ and the uniform current $\nu_{A}$.

Theorem 13.6. Let $F=F(A)$ be a finitely generated nonabelian free group. Let $\mu_{A}$ be the uniform measure on $\partial F$ corresponding to $A$. Then there exists a set $R \subseteq \partial F$ with the following properties: 
(1) We have $\mu_{A}(R)=1$.

(2) For each $\xi \in R$ there is $N \geq 1$ such that for every $n \geq N$ the element $\xi_{A}(n) \in F$ fills $F$.

Proof. Let $\nu_{A}$ be the uniform current on $F$ corresponding to $A$. Note that by construction $\nu_{A}$ has full support. As shown in [Ka4], there exists a subset $R \subseteq \partial F$ with $\mu_{A}(R)=1$ such that for each $\xi \in R$ we have

$$
\lim _{n \rightarrow \infty} \frac{\eta_{\xi_{A}(n)}}{n}=\nu_{A} \text {. }
$$

Corollary 13.3 implies that for every $\xi \in R$ there is $N \geq 1$ such that for each $n \geq N$ the element $\xi_{A}(n)$ fills $F$, as required.

Theorem 13.6 says that an "almost generic" element of $F(A)$ fills $F$.

Definition 13.7. We say that nontrivial elements $g, h \in F$ are boundedly translation equivalent in $F$, denoted $g \equiv_{b} h$, if there is $C \geq 1$ such that for every free and discrete action of $F$ on an $\mathbb{R}$-tree $T$ we have

$$
\frac{1}{C}\|h\|_{T} \leq\|g\|_{T} \leq C\|h\|_{T} .
$$

Note that in the above definition we can replace "every free and discrete action" by "every very small action".

Proposition 13.8. Let $f, g \in F$ both fill $F$. Then $f \equiv_{b} g$ in $F$.

Proof. Consider the following function $D: C V(F) \cup \partial C V(F) \rightarrow \mathbb{R}$. For every very small action of $F$ on an $\mathbb{R}$-tree $T$ put

$$
D([T]):=\frac{\|f\|_{T}}{\|g\|_{T}} .
$$

Since $f$ and $g$ both fill $F$, the function $D$ is well-defined on $C V(F) \cup \partial C V(F)$ and, moreover, $D([T])>0$ for every $[T] \in C V(F) \cup \partial C V(F)$. It is also clear that $D$ is continuous. Since $C V(F) \cup \partial C V(F)$ is compact, it follows that $D$ achieves a positive minimum and a positive maximum on $C V(F) \cup \partial C V(F)$. This implies that $f \equiv_{b} g$, as claimed.

Theorem 13.6 and Proposition 13.8 show that the phenomenon of bounded translation equivalence is "almost generic" in $F(A)$.

\section{References}

[B] H. BAss, Covering theory for graphs of groups, J. Pure Appl. Algebra 89:1-2 (1993), 3-47.

[BeF1] M. Bestvina, M. Feighn, The topology at infinity of Out $\left(F_{n}\right)$, Invent. Math. 140:3 (2000), 651-692.

[BeF2] M. Bestvina, M. Feighn, A hyperbolic Out $\left(F_{n}\right)$ complex, preprint (2008); arXiv:0808.3730

[BeF3] M. Bestvina, M. Feighn, Outer limits, preprint (1993); http://andromeda.rutgers.edu/ ${ }^{\sim}$ feighn/papers/outer.pdf

[BeFH1] M. Bestvina, M. Feighn, M. Handel, Laminations, trees, and irreducible automorphisms of free groups, Geom. Funct. Anal. 7:2 (1997), 215-244. 
[BeFH2] M. Bestvina, M. Feighn, M. Handel, The Tits alternative for $\operatorname{Out}\left(F_{n}\right)$. I. Dynamics of exponentially-growing automorphisms, Ann. of Math. (2) 151:2 (2000), 517-623.

[BeFH3] M. Bestvina, M. Feighn, M. Handel, The Tits alternative for $\operatorname{Out}\left(F_{n}\right)$. II. A Kolchin type theorem, Ann. of Math. (2) 161:1 (2005), 1-59.

[BeH] M. Bestvina, M. Handel, Train tracks and automorphisms of free groups, Ann. of Math. (2) 135:1 (1992), 1-51.

[Bo1] F. Bonahon, Bouts des variétés hyperboliques de dimension 3, Ann. of Math. (2) $124: 1$ (1986), 71-158.

[Bo2] F. Bonahon, The geometry of Teichmüller space via geodesic currents, Invent. Math. 92:1 (1988), 139-162.

[Bo3] F. Bonahon, Geodesic currents on negatively curved groups, Arboreal Group Theory (Berkeley, CA, 1988), Math. Sci. Res. Inst. Publ., 19, Springer, New York (1991), 143-168.

[CF] D. Calegari, K. Fujiwara, Combable functions, quasimorphisms, and the central limit theorem, preprint; http://arxiv.org/abs/0805.1755

[CoL] M. Cohen, M. Lustig, Very small group actions on $R$-trees and Dehn twist automorphisms, Topology 34:3 (1995), 575-617.

[Coo] D. CoOper, Automorphisms of free groups have finitely generated fixed point sets, J. Algebra 111:2 (1987), 453-456.

[CouHl1] T. Coulbois, A. Hilion, M. Lustig, $\mathbb{R}$-trees and laminations for free groups I: Algebraic laminations, J. Lond. Math. Soc. (2) 78:3 (2008), 723-736.

[CouHL2] T. Coulbois, A. Hilion, M. Lustig, $\mathbb{R}$-trees and laminations for free groups II: The dual lamination of an $\mathbb{R}$-tree, J. Lond. Math. Soc. (2) 78:3 (2008), 737-754.

[CouHl3] T. Coulbois, A. Hilion, M. Lustig, $\mathbb{R}$-trees and laminations for free groups III: Currents and dual $\mathbb{R}$-tree metrics, J. Lond. Math. Soc. (2) 78:3 (2008), 755766.

$[\mathrm{CuV}]$ M. Culler, K. Vogtmann, Moduli of graphs and automorphisms of free groups, Invent. Math. 84:1 (1986), 91-119.

[GJLL] D. Gaboriau, A. Jaeger, G. Levitt, M. Lustig, An index for counting fixed points of automorphisms of free groups, Duke Math. J. 93:3 (1998), 425-452.

[GyH] E. Ghys, P. DE la HARPe (EDs.), Sur les groupes hyperboliques d'aprés Mikhail Gromov, Birkhäuser, Progress in Mathematics 83 (1990).

[Gu] V. Guirardel, Approximations of stable actions on $R$-trees, Comment. Math. Helv. 73:1 (1998), 89-121.

[F] S. Francaviglia, Geodesic currents and length compactness for automorphisms of free groups, Trans. Amer. Math. Soc. 361:1 (2009), 161-176.

[H] U. HAMENSTÄDT, subgroups of $\operatorname{Out}\left(F_{n}\right)$, a talk at the workshop "Discrete Groups and Geometric Structures", Kortrijk, Belgium, May 2008.

[KKS] V.Kaimanovich, I. Kapovich, P. Schupp, The subadditive ergodic theorem and generic stretching factors for free group automorphisms, Israel J. Math. 157 (2007), 1-46.

[Ka1] I. KAPOVICH, Quasiconvexity and amalgams, Int. J. Alg. Comput. 7:6 (1997), $771-811$.

[Ka2] I. KAPOVICH, The combination theorem and quasiconvexity, Intern. J. Alg. Comput. 11:2 (2001), 185-216.

[Ka3] I. KaPovich, The frequency space of a free group, Internat. J. Alg. Comput. 15:5-6 (2005), 939-969. 
[Ka4] I. Kapovich, Currents on free groups, Topological and Asymptotic Aspects of Group Theory (R. Grigorchuk, M. Mihalik, M. Sapir, Z. Sunik, eds.), AMS Contemporary Mathematics Series, 394 (2006), 149-176.

[Ka5] I. KAPOVICH, Clusters, currents and Whitehead's algorithm, Experimental Mathematics 16:1 (2007), 67-76.

[KaLSS] I. Kapovich, G. Levitt, P. Schupp, V. Shpilrain, Translation equivalence in free groups, Transact. Amer. Math. Soc. 359:4 (2007), 1527-1546.

[KaLu1] I. Kapovich, M. Lustig, The actions of $\operatorname{Out}\left(F_{k}\right)$ on the boundary of outer space and on the space of currents: minimal sets and equivariant incompatibility, Ergodic Theory Dynam. Systems 27:3 (2007), 827-847.

[KaLu2] I. Kapovich, M. Lustig, Geometric intersection number and analogues of the curve complex for free groups, Geom. Topol. 13 (2009), 1805-1833.

[KaM] I. Kapovich, A. Myasnikov, Stallings foldings and the subgroup structure of free groups, J. Algebra 248:2 (2002), 608-668.

[KaN] I. Kapovich, T. Nagnibeda, The Patterson-Sullivan embedding and minimal volume entropy for outer space, Geom. Funct. Anal. 17:4 (2007), 1201-1236.

[Kap] M. Kapovich, Hyperbolic Manifolds and Discrete Groups, Birkhäuser, 2001.

[L] C.J. Leininger, Equivalent curves in surfaces, Geom. Dedicata 102 (2003), 151177.

[Le] D. LEE, Translation equivalent elements in free groups, J. Group Theory 9:6 (2006), 809-814.

[LevL] G. LEvitT, M. Lustig, Irreducible automorphisms of $F_{n}$ have north-south dynamics on compactified outer space, J. Inst. Math. Jussieu 2:1 (2003), 59-72.

[Lu] M. Lustig, A generalized intersection form for free groups, preprint (2004).

[M] R. Martin, Non-Uniquely Ergodic Foliations of Thin Type, Measured Currents and Automorphisms of Free Groups, PhD Thesis, 1995.

[P] F. Paulin, The Gromov topology on $R$-trees, Topology Appl. $32: 3$ (1989), 197221.

[S] J.-P. Serre, Trees. Springer-Verlag, Berlin-New York, 1980.

[Sh] R. Sharp, Distortion and entropy for automorphisms of free groups, Discr. Contin. Dynam. Syst. 26:1 (2010), 347-363.

[Sk] R. Skora, Deformations of length functions in groups, preprint, Columbia University (1989).

[St] M. Steiner, Gluing Data and Group Actions on $\mathbb{R}$-Trees, Thesis, Columbia University (1988).

[V] K. Vogtmann, Automorphisms of free groups and outer space, Geometriae Dedicata 94 (2002), 1-31.

Ilya Kapovich, Department of Mathematics, University of Illinois at Urbana-Champaign, 1409 West Green Street, Urbana, IL 61801, USA

kapovich@math.uiuc.edu www. math.uiuc.edu/ ${ }^{\text {kapovich/ }}$

Martin Lustig, Mathématiques (LATP), Université Paul Cézanne -Aix Marseille III, av. Escadrille Normandie-Niémen, 13397 Marseille 20, France

Martin.Lustig@univ-cezanne.fr

Received: July 30, 2008

Revision: February 20, 2009

Accepted: February 23, 2009 